\title{
On the impact of dimension-eight SMEFT operators on Higgs measurements
}

\author{
Chris Hays, ${ }^{a}$ Adam Martin, ${ }^{b}$ Verónica Sanz ${ }^{c}$ and Jack Setford ${ }^{c}$ \\ ${ }^{a}$ Department of Physics, University of Oxford, \\ Oxford OX1 3RH, U.K. \\ ${ }^{b}$ Department of Physics, University of Notre Dame, \\ Notre Dame, IN, 46556, U.S.A. \\ ${ }^{c}$ Department of Physics and Astronomy, University of Sussex, \\ Brighton BN1 9QH, U.K. \\ E-mail: chris.hays@physics.ox.ac.uk, amarti41@nd.edu, \\ v.sanz@sussex.ac.uk, j.setford@sussex.ac.uk
}

ABSTRACT: Using the production of a Higgs boson in association with a $W$ boson as a test case, we assess the impact of dimension- 8 operators within the context of the Standard Model Effective Field Theory. Dimension-8-SM-interference and dimension-6-squared terms appear at the same order in an expansion in $1 / \Lambda$, hence dimension- 8 effects can be treated as a systematic uncertainty on the new physics inferred from analyses using dimension- 6 operators alone. To study the phenomenological consequences of dimension- 8 operators, one must first determine the complete set of operators that can contribute to a given process. We accomplish this through a combination of Hilbert series methods, which yield the number of invariants and their field content, and a step-by-step recipe to convert the Hilbert series output into a phenomenologically useful format. The recipe we provide is general and applies to any other process within the dimension $\leq 8$ Standard Model Effective Theory. We quantify the effects of dimension- 8 by turning on one dimension- 6 operator at a time and setting all dimension- 8 operator coefficients to the same magnitude. Under this procedure and given the current accuracy on $\sigma\left(p p \rightarrow h W^{+}\right)$, we find the effect of dimension- 8 operators on the inferred new physics scale to be small, $\mathcal{O}$ (few $\%$ ), with some variation depending on the relative signs of the dimension- 8 coefficients and on which dimension- 6 operator is considered. The impact of the dimension- 8 terms grows as $\sigma\left(p p \rightarrow h W^{+}\right)$is measured more accurately or (more significantly) in high-mass kinematic regions. We provide a FeynRules implementation of our operator set to be used for further more detailed analyses.

Keywords: Phenomenological Models

ARXIV EPRINT: 1808.00442 


\section{Contents}

1 Introduction and motivation 1

2 Dimension-8 operator set 4

$\begin{array}{lll}2.1 & \text { Operators with zero or one derivative } & 7\end{array}$

2.1.1 Example: $\left(H^{\dagger} H\right)^{2} B_{L}^{2} \quad 7$

2.2 Operators with two or more derivatives 11

2.2.1 Example: $2 D^{2}\left(H^{\dagger} H B_{L} W_{L}\right) \quad 11$

3 Dimension-8 operators relevant for $p p \rightarrow h W \quad 15$

4 Application: $p p \rightarrow h W^{ \pm} \quad 17$

5 Conclusions 23

A Implementation in FeynRules $\quad 25$

B Dimension-6 operators $\quad 25$

C Other examples $\quad 25$

C.1 Example: $2\left(H^{\dagger} H\right)^{2} W_{L}^{2} \quad 26$

C.2 Example: $4 D\left(Q^{\dagger} Q\left(H^{\dagger} H\right)^{2}\right) \quad 27$

D Electroweak inputs and field redefinitions $\quad 28$

E Explicit form for $\bar{q} q W, h W W$ and $\bar{q} q W h$ form factors 33

F Dimension-8 effects on other operators: $c_{H Q}^{(3)}$ and $c_{H \square} \quad 33$

\section{Introduction and motivation}

The particle physicist's dream - direct detection of high-scale physics beyond the Standard Model (BSM) - has yet to be realised. As we transition into an era of precision Higgs measurements, an appropriate framework for testing the Standard Model (SM) is to treat it as an Effective Field Theory (EFT). From an EFT perspective, the SM Lagrangian is merely the first few terms in the (infinite) series:

$$
\mathcal{L}=\sum_{d} \sum_{i} \frac{c_{d}^{(i)}}{\Lambda^{4-d}} \mathcal{O}_{d}^{(i)},
$$

where $\Lambda$ is the cutoff of the effective theory, $c_{d}^{(i)}$ are the Wilson coefficients, and $\mathcal{O}_{d}^{(i)}$ are all the gauge-invariant operators at mass-dimension $d$ involving the Standard Model fields. 
The direct observation of new physics might be beyond the reach of the LHC, but it could still manifest indirectly as contributions to the Wilson coefficients of the effective theory. Constraining these coefficients is a powerful and general way to probe BSM models, since any weakly-coupled high-mass state can be integrated out to give a particular pattern of coefficient values.

The current state of the art is the classification and study of dimension-6 operators [1-4]. Several different non-redundant bases have been constructed [5-9], as have dictionaries that allow translation between them [10, 11]. Via detailed phenomenological studies and global fits, many of the Wilson coefficients of these operators have been constrained [12-39]. Some progress has also been made at dimension-8, particularly in the gauge sector [40-42].

Representing the scale at which the new physics appears as $\Lambda$, one can schematically write the amplitude for a given process as

$$
\mathcal{A} \sim\left(A_{\mathrm{SM}}+\frac{A_{\mathrm{dim}-6}}{\Lambda^{2}}+\ldots\right),
$$

where $A_{\mathrm{SM}}$ stands for the Standard Model amplitude, and $A_{\mathrm{dim}-6}$ is the correction coming from the dimension-6 EFT. The leading-order correction to the cross-section, at $\mathcal{O}\left(1 / \Lambda^{2}\right)$, is therefore an interference term of the form $A_{\mathrm{SM}} \times A_{\text {dim- } 6}$. We also have an $\left|A_{\text {dim- } 6}\right|^{2}$ correction at order $\mathcal{O}\left(1 / \Lambda^{4}\right)$, which can be readily computed with current dimension- 6 technology. However, consistent power-counting would require that, alongside $\left|A_{\text {dim- } 6}\right|^{2}$ terms, we include $A_{\mathrm{SM}} \times A_{\text {dim-8 }}$ interference effects, where now:

$$
\mathcal{A} \sim\left(A_{\mathrm{SM}}+\frac{A_{\mathrm{dim}-6}}{\Lambda^{2}}+\frac{A_{\mathrm{dim}-8}}{\Lambda^{4}}+\ldots\right) .
$$

In other words, $A_{\mathrm{SM}} \times A_{\text {dim-8 }}$ interference and $\left|A_{\text {dim- } 6}\right|^{2}$ terms both appear at $\mathcal{O}\left(1 / \Lambda^{4}\right)$, and so naively should be given equal consideration. Given that so much progress has already been made at dimension- 6 , it is natural to ask whether complementing our analyses with dimension- 8 operators can have any effect on current constraints.

The are two distinct scenarios one might consider:

1. The new physics is dominated by $A_{\mathrm{SM}} \times A_{\mathrm{dim}-6}$, i.e. interference terms between dimension- 6 contributions and the Standard Model. This is what one would naively expect whenever the scale of new physics is high in comparison with the electroweak scale.

2. The leading effect of new physics is instead $\left|A_{\text {dim- } 6}\right|^{2}$, potentially of the same order as the $A_{\mathrm{SM}} \times A_{\text {dim-8 }}$ interference terms. This situation could arise due to an accidental suppression, or indicate an underlying structure in the BSM context. For example, the interference could be suppressed by ratios of the weak scale to the cutoff $\Lambda$ to some power. One way this can occur is if there is a helicity mismatch between the SM and dimension-6 operators [43]. Another possibility is if the dimension- 6 operators are purely $\mathrm{CP}$-odd and the observable is a CP-even quantity. Or, when focusing on specific kinematic regimes, the SM content could be small and lead to the dominance of $\left|A_{\text {dim- } 6}\right|^{2}$ effects on a particular experimental bin. 
In each of these cases, we would like to know whether including dimension- 8 operators can significantly alter the bounds we put on the dimension- 6 Wilson coefficients. It may prove that an analysis purely at the dimension- 6 level is inadequate, or that dimension- 8 effects should be accounted for as a systematic uncertainty.

Constructing a complete set of effective operators at any given order is not a trivial task. It is well known that including every operator allowed by the symmetries of the theory results in an over-complete set: operators will be related to others via the equations of motion (EOM) and/or integration by parts (IBP). As discussed and illustrated in refs. [44, 45], the task is facilitated by a mathematical tool known as the Hilbert series. Given a set of objects transforming in representations of the symmetry group, the Hilbert series generates all polynomials of the objects - incorporating the symmetry (or antisymmetry) under the interchange of identical objects - and projects out all invariants using character orthonormality. Hilbert series techniques have found use in a number of theoretical [46-62] and phenomenological [4, 44, 45, 63-67] contexts. Applied to the SMEFT, the relevant symmetry group for the Hilbert series is the Lorentz symmetry group plus the $\mathrm{SU}(3) \otimes \mathrm{SU}(2) \otimes \mathrm{U}(1)$ gauge groups, and the objects entering the Hilbert series are the SM fields $\left\{Q, u^{c}, d^{c}, L, e^{c}, H\right\}$ plus the gauge field strengths. As character orthonormality only strictly applies to compact groups, we work with the Euclideanized Lorentz symmetry $\mathrm{SO}(4) \cong \mathrm{SU}(2)_{L} \otimes \mathrm{SU}(2)_{R}$. Furthermore, to correctly incorporate IBP redundancies we place all objects into representations of the conformal $\mathrm{SO}(4,2)$ group instead of the Lorentz group. The conformal representations package a field and all of its derivatives into a single object, ${ }^{1}$ and we remove the IBP redundancy from any product of objects by projecting out the highest weight component (again via character orthonormality, with some minor modifications due to the non-compact nature of $\mathrm{SO}(4,2)[4,62])$.

In the SMEFT it is natural to order the invariants by their mass dimension. The output of the Hilbert series is the number of invariants at each mass dimension, and the field content of the invariants. For example, applying the Hilbert series to the SMEFT, one of the dimension- 6 invariants is

$$
2 D^{2}\left(H^{\dagger} H\right)^{2}
$$

indicating that there are two operators containing two derivatives and four Higgs fields. What the Hilbert series does not tell us is how the various indices carried by each of the fields should be contracted. We must manually translate the output of the Hilbert series into a format that is useful for calculating Feynman rules, or for inputting into computational tools such as FeynRules [68-70]. In the case of the operator in eq. (1.4), we need to work out which fields the derivatives act on and how the Lorentz and $\mathrm{SU}(2)_{w}$ indices are contracted.

Converting the Hilbert series output into a more familiar form is one of the goals of this paper. The full set of 993 dimension-8 SMEFT operators ${ }^{2}$ is listed in ref. [4] and

\footnotetext{
${ }^{1}$ By placing scalars, fermions, and field strengths into short representations of $\mathrm{SO}(4,2)$ we can remove equation of motion (EOM) redundancies [4].

${ }^{2}$ The value of 993 holds for $N_{f}=1$, where $N_{f}$ is the number of generations, counting operators and their hermitian conjugates as separate operators. For three generations the number of dimension- 8 SMEFT operators jumps to 44807 [4]. See section 2.1 for more information.
} 


\begin{tabular}{|c|cc|c|}
\hline$Q$ & $\left(\frac{1}{2}, 0 ; 3, \frac{1}{2}, \frac{1}{6}\right)$ & $H$ & $\left(0,0 ; 0, \frac{1}{2}, \frac{1}{2}\right)$ \\
$u^{c}$ & $\left(\frac{1}{2}, 0 ; \overline{3}, 0,-\frac{2}{3}\right)$ & $B_{L}$ & $(1,0 ; 0,0,0)$ \\
$d^{c}$ & $\left(\frac{1}{2}, 0 ; \overline{3}, 0, \frac{1}{3}\right)$ & $W_{L}$ & $(1,0 ; 0,1,0)$ \\
$L$ & $\left(\frac{1}{2}, 0 ; 0, \frac{1}{2},-\frac{1}{2}\right)$ & $G_{L}$ & $(1,0 ; 8,0,0)$ \\
$e^{c}$ & $\left(\frac{1}{2}, 0 ; 0,0,1\right)$ & & \\
\hline
\end{tabular}

Table 1. Representations used in the Hilbert series construction. Hermitian conjugate representations have $\mathrm{SU}(2)_{L} \leftrightarrow \mathrm{SU}(2)_{R}$ and all gauge representations replaced by their conjugates (when applicable).

available in Mathematica format. While the recipe we outline in the following will work for all operators of any mass dimension, in this paper we explicitly work out the conversion from Hilbert series output to operators for only a subset of dimension- 8 operators. It is our hope that the recipe and examples provided will facilitate the extraction of the remaining operators for the purposes of more general and in-depth analyses. Looking to phenomenology for guidance, we have chosen to illustrate the procedure for operators which contribute to $p p \rightarrow h W$. This process is a good choice for several reasons. First, it is a relatively clean process that can be measured at high $Q^{2}$, where higher dimensional operators become important $[71,72]$. Second, it severely restricts the number of relevant dimension- 8 operators. After translating the set of required operators, we investigate $p p \rightarrow h W$, exploring how constraints on dimension-6 Wilson coefficients are affected by the inclusion of dimension- 8 effects. Our analysis focusses on one specific example but in principle one can use this technology to study many other processes, for example a Higgs boson produced in association with top quarks, or through vector boson fusion. To this end we also make publicly available the FeynRules implementation of this set of operators, so that future work can extend our analysis.

\section{Dimension-8 operator set}

The SMEFT inputs to the Hilbert series are the matter fields $\left\{Q, u^{c}, d^{c}, L, e^{c}, H\right\}$, their hermitian conjugates, and the field strength tensors. We take all fermions to be left-handed and work with combinations of the field strengths and their duals $X_{L, R}^{\mu \nu}=\frac{1}{2}\left(X^{\mu \nu} \mp i \widetilde{X}^{\mu \nu}\right)$, where $X=B, W, G$ for hypercharge, $\mathrm{SU}(2)_{w}$ and $\mathrm{SU}(3)_{c}$ respectively, since they have simpler Lorentz transformation properties $\left(X_{L} \sim(1,0), X_{R} \sim(0,1)\right.$ under $\left.\mathrm{SU}(2)_{L} \otimes \mathrm{SU}(2)_{R}\right)$. We list the full set of SMEFT representations in table 1 using the convention $\left(\operatorname{SU}(2)_{L}\right.$, $\left.\mathrm{SU}(2)_{R} ; \mathrm{SU}(3)_{c}, \mathrm{SU}(2)_{w}, \mathrm{U}(1)_{Y}\right)$. The fields are dressed with characters corresponding to their gauge and conformal representations, and plugged into the Hilbert series generating function. Details of the characters, and of the reduction of the polynomial produced by the generating function to gauge and Lorentz singlets, can be found in refs. [4, 44, 45]. Weighting each operator in the output by its mass dimension, we can easily filter out the dimension- 8 operators. 
As mentioned in the introduction, we have chosen to illustrate the procedure for operators that can contribute to $p p \rightarrow h W$ processes. Following the power-counting argument in eq. (1.3), we are interested in the subset of dimension-8 operators that can interfere with the SM contributions to $p p \rightarrow h W$. If we take all first and second generation fermions to be massless, ${ }^{3}$ the leading-order SM $p p \rightarrow h W$ amplitudes all proceed through a single topology: the process is initiated by a left-handed quark and an antiquark that annihilate into a $W$ boson, which then emits a Higgs boson. The requirement that dimension- 8 operators interfere with this amplitude limits us to three types of operator: ${ }^{4} 1$.) operators that modify the $h W W$ vertex, 2.) operators that modify the $\bar{q} q W$ vertex, and 3.) four-point contact operators. The relevant Feynman diagrams are shown in figure 1. Operators of the first type are purely bosonic and involve Higgs fields, derivatives, and field strengths, while operators of the latter two types must involve a like-chirality fermion-antifermion pair, at most one field strength, Higgs fields and derivatives. All three operator types must be included in order to have a correct result. We neglect modifications to the $W$ and $h$ widths, and instead treat them as on-shell final-state particles.

We now extract from the Hilbert series the relevant pieces for the operators we wish to consider. Due to the way EOM are handled in the Hilbert series machinery, its output is always in the so-called Warsaw basis [5], where higher derivative terms are removed in favor of operators with more fields whenever possible. Therefore, when combining dimension- 8 and dimension- 6 effects in later sections, we use the Warsaw basis for dimension-6. For other advantages of the Warsaw basis, see refs. [43, 73, 74]. Focusing first on the bosonic operators, we can group the dimension- 8 operators according to the number of derivatives. At $\mathcal{O}\left(D^{0}\right)$, we find:

$$
\begin{aligned}
& \left(H^{\dagger} H\right)^{4}, \quad\left(H^{\dagger} H\right)^{2}\left(B_{L}\right)^{2}, \quad\left(H^{\dagger} H\right)^{2} B_{L} W_{L}, \quad 2\left(H^{\dagger} H\right)^{2}\left(W_{L}\right)^{2}, \\
& \left(H^{\dagger} H\right)^{2}\left(G_{L}\right)^{2}, \quad\left(H^{\dagger} H\right) B_{L}\left(W_{L}\right)^{2}, \quad\left(H^{\dagger} H\right)\left(W_{L}\right)^{3}, \quad\left(H^{\dagger} H\right)\left(G_{L}\right)^{3},
\end{aligned}
$$

where for all operators except $\left(H^{\dagger} H\right)^{4}$ there is a corresponding hermitian conjugate operator with $B_{L}, W_{L}, G_{L} \rightarrow B_{R}, W_{R}, G_{R}$. At $\mathcal{O}\left(D^{2}\right)$ the operators are:

$$
\begin{aligned}
& 2 D^{2}\left(H^{\dagger} H\right)^{3}, \quad D^{2}\left(H^{\dagger} H B_{L} B_{R}\right), \quad D^{2}\left(H^{\dagger} H G_{L} G_{R}\right), 2 D^{2}\left(H^{\dagger} H W_{L} W_{R}\right), \\
& D^{2}\left(H^{\dagger} H\right)^{2} B_{L}, D^{2}\left(H^{\dagger} H\left(B_{L}\right)^{2}\right), D^{2}\left(H^{\dagger} H\left(G_{L}\right)^{2}\right), 2 D^{2}\left(H^{\dagger} H\left(W_{L}\right)^{2}\right), \\
& 2 D^{2}\left(H^{\dagger} H B_{L} W_{L}\right), \quad D^{2}\left(H^{\dagger} H B_{R} W_{L}\right), \quad 2 D^{2}\left(\left(H^{\dagger} H\right)^{2} W_{L}\right) .
\end{aligned}
$$

The first row of operators are self-hermitian, while hermitian conjugates must be added for the operators in the second and third rows. Finally, at $\mathcal{O}\left(D^{4}\right)$ there is one operator set:

$$
3 D^{4}\left(H^{2} H^{\dagger 2}\right)
$$

\footnotetext{
${ }^{3}$ Also ignoring bottom and top quark components of the proton.

${ }^{4}$ Here we are referring to new vertices. Dimension- 8 operators can also indirectly enter $p p \rightarrow h W$ through field redefinitions or through the relations between couplings and experimental inputs. We will study these effects in more detail in section 4 and appendix D.
} 

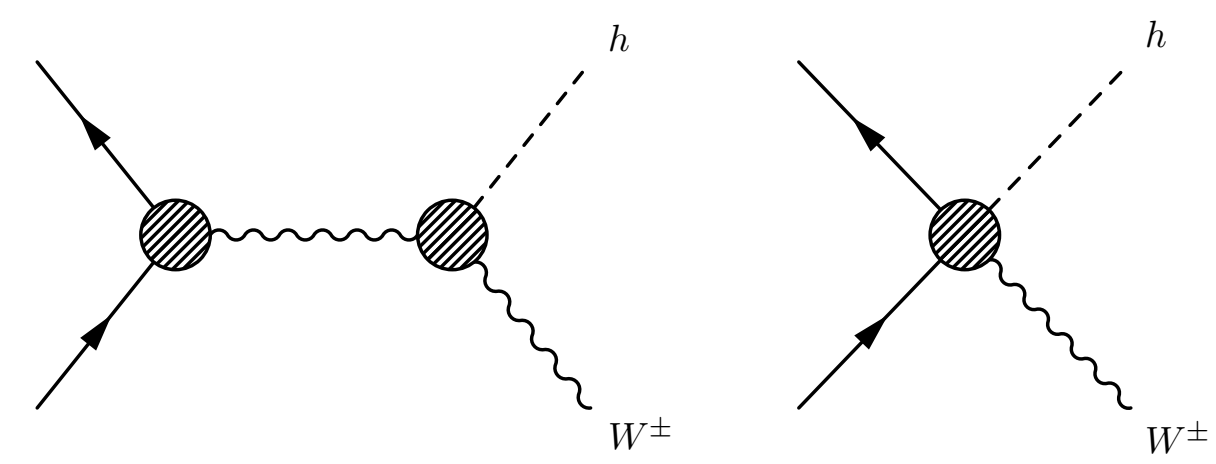

Figure 1. Diagrams contributing to $p p \rightarrow h W^{ \pm}$. The shaded circles represent integrated-out new physics, and mark the vertices modified by the HDOs we have included in our analysis. Left: associated production via an $s$-channel $W$ boson. In this case the purely bosonic operators modify the $h W W$ coupling, and the contact operators modify the quark- $W$ vertex. Right: the four-point contact interaction that can also mediate $p p \rightarrow h W^{ \pm}$.

which is self-hermitian. Moving on to the contact operators and operators that modify the $\bar{q} q W$ vertices, the Hilbert series output for left-handed quarks is:

$$
\begin{aligned}
& 4 D\left(Q^{\dagger} Q\left(H^{\dagger} H\right)^{2}\right), 6 D\left(Q^{\dagger} Q H^{\dagger} H W_{L}\right), \\
& 4 D^{3}\left(Q^{\dagger} Q H^{\dagger} H\right),
\end{aligned}
$$

with additional hermitian conjugates for the $W_{L}$ terms. The operators with no field strengths will impact the $\bar{q} q W$ couplings, and all sets will generate $\bar{q} q W h$ contact terms.

We could have written the fermionic operators with a generation index, leading to a different operator for each possible generation combination, $Q_{1}^{\dagger} Q_{3}, Q_{2}^{\dagger} Q_{1}$, etc. In some circumstances, adding generation indices would disrupt the antisymmetrization we must perform when an operator contains multiple identical fermionic fields. For these operators, there are no repeated fields, so adding generation indices would just multiply the number of operators by $N_{f}^{2}$. Throughout this paper we will ignore this complication and work with $N_{f}=1$. For operators with only two fermions, such as those in eq. (2.4), this simplification amounts to assuming that the operator effects are universal among the fermion generations, e.g. the coefficient for $\bar{u} u W h$ is the same as for $\bar{c} c W h$, etc. For operators with more than two fermions, the $N_{f}=1$ restriction is more severe as it omits any communication between generations, as e.g. $\left(Q_{1}^{\dagger} Q_{1}\right)^{2}$ is allowed but $\left(Q_{1}^{\dagger} Q_{1}\right)\left(Q_{2}^{\dagger} Q_{2}\right)$ is not. ${ }^{5}$ This fact will play a role when we discuss how higher dimensional operators impact the interpretation of experimental quantities such as $\alpha_{e m}, M_{Z}$ or $G_{F}$ in terms of theory inputs in section 4 .

As we can see, the Hilbert series tells us exactly how many independent invariants we can construct from each combination of fields, but it does not tell us how the indices should be contracted. In the next two subsections we detail how to convert this output into canonical phenomenological form. Readers more interested in the applications of dimension-8 operators can skip to section 3.

\footnotetext{
${ }^{5}$ We note that our $N_{f}=1$ restriction differs from the usual $\mathrm{U}(3)^{5}$ assumption, which has complete flavour universality and a common coupling for all four-fermion combinations. In our simplified $N_{f}=1$ framework the high-scale physics affects the four-fermion couplings only within each generation.
} 


\subsection{Operators with zero or one derivative}

For operators with zero or one derivative, the process of converting the Hilbert series output into useful phenomenological form is fairly straightforward and can be broken down into six steps:

1. if the operator contains multiple instances of the same field $(H, Q$, etc.), work out the group products that are allowed given the Bose or Fermi symmetry;

2. from the properly symmetrized blocks, determine the contractions that lead to an overall invariant for the entire operator;

3. express the contractions using the available group-theory objects, e.g. $\epsilon_{a b}$ for $\mathrm{SU}(2)$ and $\delta_{B}^{A}$ or $f_{A B C}$ for $\mathrm{SU}(3)$;

4. translate Lorentz contractions from $\mathrm{SU}(2)_{L} \otimes \mathrm{SU}(2)_{R}$ to $\mathrm{SO}(3,1)$;

5. translate from the $\mathrm{L}$ and $\mathrm{R}$ field-strength combinations to the field strength $X_{\mu \nu}$ and its dual $\tilde{X}_{\mu \nu}$;

6. impose hermiticity.

Steps 1 and 2 require picking out singlets from products of representations, thereby dictating how the various indices need to be contracted, while steps 3 through 5 take us from the formalism convenient for group theory to canonical operator conventions. We emphasize that the six steps give us the form of the operators corresponding to the Hilbert

series and not the coefficient accompanying the operator, i.e. the factor $c_{d}^{(i)}$ in eq. (1.1). The coefficients can only be set knowing the UV theory. Because of this ignorance of the operator coefficient, we will completely ignore all overall numerical (and sign) factors that appear when translating operators, subsuming them into the $c_{d}^{(i)}$.

The conversion steps are best illustrated with an example. We will work out the steps for this example by hand, though steps 1 and 2 can also be performed using programs such as susyno [75]. While it is often obvious to see how the indices on an operator must be contracted without plodding through every step, we will go through this example in full detail so that the process can be applied in cases where inspection fails. Other examples can be found in appendix C.

\subsubsection{Example: $\left(H^{\dagger} H\right)^{2} B_{L}^{2}$}

Before moving on to the operator conversion, let us define our index conventions. Most of the index manipulations will involve $\mathrm{SU}(2)$. We will use undotted Greek letters for $\mathrm{SU}(2)_{L}$ indices, dotted Greek letters for $\mathrm{SU}(2)_{R}$ indices, and Latin letters for $\mathrm{SU}(2)_{w}$. When we convert from Lorentz $\mathrm{SU}(2)_{L} \otimes \mathrm{SU}(2)_{R}$ to $\mathrm{SO}(3,1)$ we will also use Greek indices for $\mathrm{SO}(3,1)$, though drawn from the middle of the alphabet (which should be clear from the context). Symmetrized groups of indices will be indicated by curly braces $\{\alpha \beta\}$ and antisymmetric ones by brackets, $[a b]$. Occasionally we will find it convenient to convert products of $\mathrm{SU}(2)$ doublets into triplets using Pauli matrices, which will be indicated by 
upper-case Latin letters (i.e. $\tau^{I}$ ). While $\mathrm{SU}(2)$ doublet indices can only be contracted by $\epsilon_{i j},{ }^{6}$ triplet indices may be contracted by $\delta^{I J}$ or the antisymmetric $\epsilon_{I J K}$. As an example, the singlet contraction of the $\mathrm{SU}(2)$ doublets $H^{\dagger}$ and $H$ is given by $\epsilon^{i j} H_{i}^{\dagger} H_{j}$. The form $\epsilon^{i j} H_{i}^{\dagger} H_{j}$ may seem a bit strange at first glance. It occurs because we are working with $H^{\dagger}$ (and $L^{\dagger}, Q^{\dagger}$ ) as a 2 of $\mathrm{SU}(2)$ rather than a $\overline{2}$, as this makes the group manipulation simpler. One can easily convert between the 2 and $\overline{2}$ forms via $\epsilon: H_{\overline{2}}^{\dagger}=\epsilon H_{2}^{\dagger}$. Throughout this paper we will perform all intermediate steps using $H_{2}^{\dagger}$, then convert to the more familiar $H_{\overline{2}}^{\dagger}$ form at the end.

Now we turn to our first example: $\left(H^{\dagger} H\right)^{2} B_{L}^{2}$. As the coefficient of this Hilbert series output is 1 , there is one invariant to find. The operator involves three different fields, each of which occurs twice. As $H^{\dagger} \neq H$ and $B_{L}$ are all bosonic, each block of (field) ${ }^{2}$ must be symmetric. Starting with the Higgs field and dropping the $\mathrm{SU}(3)_{c}$ and $\mathrm{U}(1)_{Y}$ entries for brevity,

$$
H^{2}=\left(0,0 ; \frac{1}{2}\right)_{\mathrm{symm}}^{2}=(0,0 ; 0 \oplus 1)_{\mathrm{symm}}=(0,0 ; 1) .
$$

This is telling us nothing more than $H_{i} H_{j}$ contracted with $\epsilon^{i j}$ gives zero (where $i, j$ are $\mathrm{SU}(2)_{w}$ indices). Only the symmetric combination $H_{\left\{i, H_{j\}}\right.}$, or $H_{\{i j\}}^{2}$, is nonzero. $\left(H^{\dagger}\right)^{2}$ is the same as $H^{2}$, so we can proceed to $B_{L}^{2}$ :

$$
B_{L}^{2}=(1,0 ; 0)_{\mathrm{symm}}^{2}=(0 \oplus 1 \oplus 2,0 ; 0)_{\mathrm{symm}}=(0 \oplus 2,0 ; 0) .
$$

This decomposition is also straightforward: the symmetric product of two spin-1 fields must be spin- 0 or spin- $2 .{ }^{7}$ Multiplying the three blocks together gives:

$$
H^{2}\left(H^{\dagger}\right)^{2} B_{L}^{2}=(0,0 ; 1) \otimes(0,0 ; 1) \otimes(0 \oplus 2,0 ; 0)=(0 \oplus 2,0 ; 0 \oplus 1 \oplus 2) .
$$

Choosing the 0 from the $\mathrm{SU}(2)_{L}$ and $\mathrm{SU}(2)_{w}$ products we get the one invariant promised by the Hilbert series. Notice we have $0 \oplus 1 \oplus 2$ in the $\mathrm{SU}(2)_{w}$ portion since there is no (anti)symmetrization left to eliminate a piece.

The above procedure takes us through step 2. For step 3, we can focus on the Higgs portion since that contains all the $\mathrm{SU}(2)_{w}$ dependence. For SU(2), the only object available to contract indices is $\epsilon^{i j}$. The contraction that does not vanish is:

$$
H_{\{i j\}}^{2}\left(H^{\dagger}\right)_{\{k m\}}^{2} \epsilon^{i k} \epsilon^{j m} .
$$

The contraction of $i$ with $m$ and $j$ with $k$ is equivalent since $i \leftrightarrow j, k \leftrightarrow m$ is symmetric, and contracting $i$ with $j$ gives zero. Written in a more familiar way, we have

$$
\left(\epsilon H^{\dagger} H\right)^{2}
$$

\footnotetext{
${ }^{6}$ We define $\epsilon^{12}=-\epsilon^{21}=\epsilon_{21}=-\epsilon_{12}=1$, following the conventions in [76].

${ }^{7}$ The reader may wonder why we are using Lorentz representations in this section after alluding to conformal representations in section 1 . Conformal representations package an object and all its derivatives together and are useful for automatically incorporating IBP and EOM redundancies [4, 62]. To explicitly construct operators for given field (and derivative) content, working with Lorentz representations is sufficient.
} 
where we have ignored any overall factors of 2 or -1 . Moving on to $\mathrm{SU}(2)_{L}, B_{L}$ sits in the triplet representation, so in terms of fundamental $\mathrm{SU}(2)_{L}$ indices it is a symmetric tensor $B_{L\{\alpha \beta\}}$. Taking the product of two $B_{L}$ fields, the $\mathrm{SU}(2)_{L}$ invariant comes from contracting the indices with $\epsilon$ :

$$
\left(B_{L}^{2}\right)_{(0,0)}=B_{L\{\alpha \beta\}} B_{L\{\gamma \delta\}} \epsilon^{\alpha \gamma} \epsilon^{\beta \delta} .
$$

To convert from this format to the more familiar $\mathrm{SO}(3,1)$ language (step 4), our starting point is the decomposition of antisymmetric tensors [77]:

$$
X^{\mu \nu}=\sigma^{\mu \alpha \dot{\alpha}} \sigma^{\nu \beta \dot{\beta}}\left(\epsilon_{\dot{\alpha} \dot{\beta}} A_{\{\alpha \beta\}}+\epsilon_{\alpha \beta} B_{\{\dot{\alpha} \dot{\beta}\}}\right),
$$

where $\mu, \nu$ are the usual $\mathrm{SO}(3,1)$ indices and undotted (dotted) $\alpha, \beta$ are $\mathrm{SU}(2)_{L}\left(\mathrm{SU}(2)_{R}\right)$ indices. We can see that $A$ sits in the $(1,0)$ representation of $\mathrm{SU}(2)_{L} \otimes \mathrm{SU}(2)_{R}$ while $B$ sits in $(0,1)$. Manipulating eq. (2.11) using the properties of $\epsilon$ and the $\sigma$ matrices [76], we find

$$
A_{\{\alpha \beta\}}=2 i\left(\sigma^{\mu \nu}\right)_{\alpha \beta} X_{L \mu \nu}, \quad B_{\{\dot{\alpha} \dot{\beta}\}}=2 i\left(\bar{\sigma}^{\mu \nu}\right)_{\dot{\alpha} \dot{\beta}} X_{R \mu \nu},
$$

where $\sigma^{\mu \nu}$ and $\bar{\sigma}^{\mu \nu}$ are antisymmetric in $\mathrm{SO}(3,1)$ indices but symmetric in either $\mathrm{SU}(2)_{L}$ or $\mathrm{SU}(2)_{R}$ indices. Applying eq. (2.12) to eq. (2.10) gives:

$$
\begin{aligned}
B_{L\{\alpha \beta\}} B_{L\{\gamma \delta\}} \epsilon^{\alpha \beta} \epsilon^{\gamma \delta} & =-4\left(\sigma^{\mu \nu}\right)_{\alpha \beta}\left(\sigma^{\rho \sigma}\right)_{\gamma \delta} \epsilon^{\alpha \gamma} \epsilon^{\beta \delta} \times B_{L \mu \nu} B_{L \rho \sigma} \\
& =-4 \operatorname{Tr}\left(\sigma^{\mu \nu} \sigma^{\rho \sigma}\right) B_{L \mu \nu} B_{L \rho \sigma} \\
& =-2\left(g^{\mu \rho} g^{\nu \sigma}-g^{\mu \sigma} g^{\nu \rho}-i \epsilon^{\mu \nu \rho \sigma}\right) B_{L \mu \nu} B_{L \rho \sigma} \\
& =-4\left(B_{L \mu \nu} B_{L}^{\mu \nu}-i B_{L \mu \nu} \tilde{B}_{L}^{\mu \nu}\right) .
\end{aligned}
$$

Next, we can convert ${ }^{8}$ from $B_{L}$ to $B$ and $\tilde{B}$, and $H_{2}^{\dagger}$ to $H_{\frac{2}{2}}^{\dagger}$, with the net result:

$$
\mathcal{O}_{H 2 B 2}=\left(H^{\dagger} H\right)^{2}\left(B_{\mu \nu} B^{\mu \nu}-i B_{\mu \nu} \tilde{B}^{\mu \nu}\right),
$$

again dropping overall numerical factors and subscripts.

Finally, we must impose hermiticity. Repeating the conversion steps on the hermitian conjugate output $\left(H^{\dagger} H\right)^{2} B_{R}^{2}$, we get (unsurprisingly) the hermitian conjugate of eq. (2.14). Including both $\mathcal{O}_{H 2 B 2}$ and $\mathcal{O}_{H 2 B 2}^{\dagger}$ into the Lagrangian, hermiticity demands that their coefficients are complex conjugates of each other,

$$
\mathcal{L} \supset c_{H 2 B 2} \mathcal{O}_{H 2 B 2}+c_{H 2 B 2}^{*} \mathcal{O}_{H 2 B 2}^{\dagger},
$$

corresponding to two real coefficients instead of four. We can regroup these terms into two separate operators involving Higgs fields and hypercharge field strengths:

$$
\mathcal{O}_{1}=\left(H^{\dagger} H\right)^{2} B_{\mu \nu} B^{\mu \nu}, \quad \mathcal{O}_{2}=\left(H^{\dagger} H\right)^{2} B_{\mu \nu} \tilde{B}^{\mu \nu} .
$$

Either in the form of eq. (2.15) or eq. (2.16), we see that the result of steps 1-6 is a number of operators matching the Hilbert series output. In eq. (2.16) the two operators corresponding

\footnotetext{
${ }^{8}$ When converting between the $X_{L, R}$ and $X, \tilde{X}$ forms, the relations $\epsilon^{\mu \nu \rho \sigma} X_{L, \rho \sigma}=2 i X_{L}^{\mu \nu}, \epsilon^{\mu \nu \rho \sigma} X_{R, \rho \sigma}=$ $-2 i X_{R}^{\mu \nu}$ are particularly handy.
} 
to the outputs $\left(H^{\dagger} H\right)^{2} B_{L, R}^{2}$ are obvious, while in eq. (2.15) they are combined into a complex, non-hermitian operator with a complex coefficient. ${ }^{9}$ In cases where there is more than one operator with a given field content, there are more options for the operators (and their combinations) to use. This can be seen with $2\left(H^{\dagger} H\right)^{2} W_{L}^{2}$, which also has nontrivial $\mathrm{SU}(2)_{w}$ contractions and is presented in appendix $\mathrm{C}$. These choices are inevitable and represent a choice of basis. Note the dimension- $8 \mathrm{CPV}$ terms will not affect cross sections at $\mathcal{O}\left(1 / \Lambda^{4}\right)$ since they have no SM piece to interfere with. We include them purely for completeness and to show how the operator counting works at different stages of the translation process.

The steps above carry over to operators with a single derivative. The new ingredient is the derivative $D$, which transforms as $\left(\frac{1}{2}, \frac{1}{2} ; 0,0,0\right)$ under the symmetry groups and is otherwise treated like any other bosonic object in an operator. When converting an operator of the form $D(A B C)$, where $A, B, C$ are SMEFT fields, the first conversion step is to partition the derivative among the fields. This is not as automatic as it seems, since i.) we are only interested in products of derivatives and fields that do not reduce by the equations of motion, and ii.) it is often the case that we cannot apply the derivative to all fields present, e.g. for $D A B C$ there may be no way to make an invariant with $A B D(C)$. The procedure for removing EOM-reducible terms in Hilbert series output was put forth in ref. [44]. In short, we treat $D \psi\left(\psi\right.$ a left-handed fermion) as a Lorentz $\left(1, \frac{1}{2}\right)$ in all products. Similarly, $D \psi^{\dagger} \sim\left(\frac{1}{2}, 1\right), D X_{L} \sim\left(\frac{3}{2}, \frac{1}{2}\right)$ and $D X_{R} \sim\left(\frac{1}{2}, \frac{3}{2}\right) .{ }^{10}$ In terms of $\mathrm{SU}(2)_{L} \times \mathrm{SU}(2)_{R}$ indices:

$$
D \psi \sim(D \psi)_{\{\alpha \beta\}, \dot{\alpha}} \quad D \psi^{\dagger} \sim\left(D \psi^{\dagger}\right)_{\alpha,\{\dot{\alpha} \dot{\beta}\}} \quad D X_{L} \sim\left(D X_{L}\right)_{\{\alpha \beta \gamma\}, \dot{\alpha}} \quad D X_{R} \sim\left(D X_{R}\right)_{\alpha,\{\dot{\alpha} \dot{\beta} \dot{\gamma}\}}
$$

and we can convert between $\mathrm{SU}(2)_{L} \times \mathrm{SU}(2)_{R}$ and $\mathrm{SO}(3,1)$ using

$$
D_{\alpha \dot{\alpha}}=D_{\mu}\left(\sigma^{\mu}\right)_{\alpha \dot{\alpha}}
$$

In the manipulations above we have only shown the Lorentz part of the representations. Once partitioned onto a field, the derivative should be thought of as a covariant derivative, so $D \psi, D X_{L}$, etc. will carry the gauge representations appropriate to $\psi, X_{L}$, etc.

Once we know which derivative partitions are allowed, we are free to pick which one to use since they are easily related to each other by integration by parts. For example, if $D(A B C)=D(A) B C+A D(B) C$ then we may pick whichever we like as our operator. Once we have chosen how to partition the derivative, we proceed with steps $1-6$ of section 2.1 to convert them into operators. There are no purely bosonic operators in the SMEFT containing a single derivative, therefore we defer an example of an $\mathcal{O}(D)$ operator (involving fermions) to appendix C.

\footnotetext{
${ }^{9}$ Said differently, the Hilbert series output dictates the number of real operator coefficients for a given field content. Customarily, bosonic operators are written in the form of eq. (2.16), with real coefficients, while fermionic operators are written in the form of eq. (2.15), with complex coefficients and an implicit addition of the hermitian conjugate.

${ }^{10}$ There is no EOM reduction for scalars at $\mathcal{O}(D)$, where $D \phi \sim\left(\frac{1}{2}, \frac{1}{2}\right)$. There is reduction at $\mathcal{O}\left(D^{2}\right)$, where $D^{2} \phi \sim(1,1)$.
} 


\subsection{Operators with two or more derivatives}

Operators containing two or more derivatives are trickier. The presence of derivatives implies that we have redundancies due to integration by parts, which can shift the covariant derivative from one field to another, and the equations of motion. We must be careful to ensure that our final result does not contain any redundancies, but has enough flexibility to generate (via IBP) any operator with the same fields and number of derivatives. As before, these issues are best demonstrated with an example.

\subsubsection{Example: $2 D^{2}\left(H^{\dagger} H B_{L} W_{L}\right)$}

Let us look at one of the classes of operators from eq. (2.2): $2 D^{2}\left(H^{\dagger} H B_{L} W_{L}\right)$. The Hilbert series tells us that there are only two independent invariants for this combination of fields and derivatives, but which two operators do we pick? At first glance there are multiple ways of placing the derivatives,

$$
\begin{aligned}
& \left(D_{\mu} H^{\dagger}\right)\left(D_{\mu} H\right) W_{L, \rho \sigma} B_{L, \rho \sigma}, \quad\left(D_{\mu} H^{\dagger}\right)\left(D^{\nu} H\right) W_{L, \mu \rho} B_{L, \rho \nu}, \\
& H^{\dagger} H\left(D_{\mu} W_{L, \nu \rho}\right)\left(D^{\mu} B_{L}^{\nu \rho}\right), \quad\left(D_{\mu} H^{\dagger}\right) H\left(D_{\mu} W_{L, \nu \rho}\right) B_{L, \nu \rho}, \quad \ldots
\end{aligned}
$$

If all operators were equal, we could just pick any two. However, this is not the case. After picking one operator from eq. (2.18) there are some choices for the second operator which - combined with the original operator - can transform via IBP into any of the other $D^{2}\left(H^{\dagger} H B_{L} W_{L}\right)$ operators, while there are other operators that will only transform into a subset. We need to pick two operators that any of the possible $D^{2}\left(H^{\dagger} H B_{L} W_{L}\right)$ operators can be reduced to by successive IBP. In order to make the right choice in this example and in similar cases, we need to know how IBP relates all operators with a given field and derivative content.

To systematically understand the IBP relations and their use in reducing the number of operators, we will follow the approach described in ref. [45]. Our first step is to enumerate the ways to partition the derivatives, as partially illustrated in eq. (2.18). Given our previous experience with adding indices, we can immediately recognize that i.) after removing EOM-reducible terms, $D^{2} H^{\dagger}$ and $D^{2} H$ (both transforming as $(1,1)$ under the Lorentz group) will never admit a Lorentz singlet since all other fields only transform under Lorentz $\mathrm{SU}(2)_{L}$, and ii.) the $\mathrm{SU}(2)_{w}$ part of the index contraction is trivial, as $H_{i}^{\dagger} H_{j}$ must form a triplet to contract with $W_{L}$. Partitioning the derivatives all possible ways, there are seven different operators in $D^{2}\left(H^{\dagger} H B_{L} W_{L}\right)$. The operators are listed in table 2 with $\mathrm{SU}(2)_{w}$ indices suppressed.

The first two operators correspond to the two ways we can pair $\left(D H^{\dagger} D H\right) \supset(0 \oplus 1,0 \oplus$ $1 ; 0,0 \oplus 1,0)$ with $B_{L} W_{L} \supset(0 \oplus 1 \oplus 2,0 ; 0,1,0) .{ }^{11}$ We will stick to the $\mathrm{SU}(2)_{L} \otimes \mathrm{SU}(2)_{R}$ form of Lorentz symmetry throughout to avoid translating derivatives of field strengths

\footnotetext{
${ }^{11}$ In the first operator the Lorentz indices of $D H^{\dagger} D H$ are stitched together to form a singlet, as are the indices of $B_{L} W_{L}$. In the second operator, we pick out the $(1,0)$ part of $B_{L} W_{L}$ by contracting one index on each field strength together, then combine that object with the $(1,0)$ piece of $D H^{\dagger} D H$. The two operators represent the two different ways to tie the indices of $D H^{\dagger} D H$ to the indices of $B_{L} W_{L}$; we could collapse the two operators to one (plus a piece looking like $x_{1}$ ) via the Schouten identity, but the current form makes the algebra easier.
} 


\begin{tabular}{|c|c|}
\hline$x_{1}$ & $\left(D H^{\dagger}\right)_{\alpha \dot{\alpha}}(D H)_{\beta \dot{\beta}} B_{L\{\gamma \delta\}} W_{L\{\xi \eta\}} \epsilon^{\dot{\alpha} \dot{\beta}} \epsilon^{\alpha \beta} \epsilon^{\gamma \xi} \epsilon^{\delta \eta}$ \\
$x_{2}$ & $\left(D H^{\dagger}\right)_{\alpha \dot{\alpha}}(D H)_{\beta \dot{\beta}} B_{L\{\gamma \delta\}} W_{L\{\xi \eta\}} \frac{1}{2} \epsilon^{\dot{\alpha} \dot{\beta}} \epsilon^{\delta \xi}\left(\epsilon^{\alpha \gamma} \epsilon^{\beta \eta}+\epsilon^{\beta \gamma} \epsilon^{\alpha \eta}\right)$ \\
$x_{3}$ & $\left(D H^{\dagger}\right)_{\alpha \dot{\alpha}} H\left(D B_{L}\right)_{\{\beta \gamma \delta\}, \dot{\beta}} W_{L\{\xi \eta\}} \epsilon^{\dot{\alpha} \dot{\beta}} \epsilon^{\alpha \beta} \epsilon^{\gamma \xi} \epsilon^{\delta \eta}$ \\
$x_{4}$ & $\left(D H^{\dagger}\right)_{\alpha \dot{\alpha}} H B_{L\{\xi \eta\}}\left(D W_{L}\right)_{\{\beta \gamma \delta\}, \dot{\beta}} \epsilon^{\dot{\alpha} \dot{\beta}} \epsilon^{\alpha \beta} \epsilon^{\gamma \xi} \epsilon^{\delta \eta}$ \\
$x_{5}$ & $H^{\dagger}(D H)_{\alpha \dot{\alpha}}\left(D B_{L}\right)_{\{\beta \gamma \delta\}, \dot{\beta}} W_{L\{\xi \eta\}} \epsilon^{\dot{\alpha} \dot{\beta}} \epsilon^{\alpha \beta} \epsilon^{\gamma \xi} \epsilon^{\delta \eta}$ \\
$x_{6}$ & $H^{\dagger}(D H)_{\alpha \dot{\alpha}} B_{L\{\xi \eta\}}\left(D W_{L}\right)_{\{\beta \gamma \delta\}, \dot{\beta}} \epsilon^{\dot{\alpha} \dot{\beta}} \epsilon^{\alpha \beta} \epsilon^{\gamma \xi} \epsilon^{\delta \eta}$ \\
$x_{7}$ & $H^{\dagger} H\left(D B_{L}\right)_{\{\alpha \beta \gamma\}, \dot{\alpha}}\left(D W_{L}\right)_{\{\xi \eta \delta\}, \dot{\beta}} \epsilon^{\dot{\alpha} \dot{\beta}} \epsilon^{\alpha \xi} \epsilon^{\beta \eta} \epsilon^{\gamma \delta}$ \\
\hline
\end{tabular}

Table 2. Operators of the type $D^{2}\left(H^{\dagger} H B_{L} W_{L}\right)$ where we have ignored IBP relations between terms. We have neglected all $\mathrm{SU}(2)_{w}$ indices since there is only one possible contraction.

\begin{tabular}{|c|c|}
\hline$y_{1}$ & $\left(D H^{\dagger}\right)_{\alpha \dot{\alpha}} H B_{L\{\beta \gamma\}} W_{L\{\xi \eta\}} \epsilon^{\beta \xi} \epsilon^{\gamma \eta}$ \\
$y_{2}$ & $\left(D H^{\dagger}\right)_{\alpha \dot{\alpha}} H B_{L\{\beta \gamma\}} W_{L\{\xi \eta\}} \frac{1}{2} \epsilon^{\gamma \xi}\left(\epsilon^{\alpha \beta}+\epsilon^{\alpha \eta}\right)$ \\
$y_{3}$ & $H^{\dagger}(D H)_{\alpha \dot{\alpha}} B_{L\{\beta \gamma\}} W_{L\{\xi \eta\}} \epsilon^{\beta \xi} \epsilon^{\gamma \eta}$ \\
$y_{4}$ & $H^{\dagger}(D H)_{\alpha \dot{\alpha}} B_{L\{\beta \gamma\}} W_{L\{\xi \eta\}} \frac{1}{2} \epsilon^{\gamma \xi}\left(\epsilon^{\alpha \beta}+\epsilon^{\alpha \eta}\right)$ \\
$y_{5}$ & $H^{\dagger} H\left(D B_{L}\right)_{\{\alpha \beta \gamma\}, \dot{\alpha}} W_{L\{\xi \eta\}} \epsilon^{\beta \xi} \epsilon^{\gamma \eta}$ \\
$y_{6}$ & $H^{\dagger} H B_{L\{\xi \eta\}}\left(D W_{L}\right)_{\{\alpha \beta \gamma\}, \dot{\alpha}} \epsilon^{\beta \xi} \epsilon^{\gamma \eta}$ \\
\hline
\end{tabular}

Table 3. Operators of the type $D\left(H^{\dagger} H B_{L} W_{L}\right)$ that are gauge-invariant but sit in the Lorentz four-vector representation. The number of operators in this class can be generated automatically via the same procedure that projects out the number of total invariants. As in table 2, we have suppressed $\mathrm{SU}(2)_{w}$ indices.

into $\mathrm{SO}(3,1)$ language. When contracting indices we have made a choice of the overall sign. Nothing will depend on this choice, but we do need to be careful to stick with this convention.

Next, we need the set of operators with one less derivative, $D\left(H^{\dagger} H B_{L} W_{L}\right)$ that sits in the four-vector Lorentz representation. The group theory here follows exactly as before, except that we are picking products in the $\left(\frac{1}{2}, \frac{1}{2}\right)$ representation rather than Lorentz singlets. The single derivative can act on each of the four fields, $D H^{\dagger} \cdots, H^{\dagger} D H \cdots, H^{\dagger} H(D B)$, etc., and for the $D H^{\dagger}, D H$ options there are two ways to form $\left(\frac{1}{2}, \frac{1}{2}\right)$. Working this out generates the six $D\left(H^{\dagger} H B_{L} W_{L}\right)$ terms in table 3 .

The relation between the $y_{i}$ - gauge invariant operators with one fewer derivative and sitting in the four-vector Lorentz representation - and IBP is now easy to see. Contracting any of the $y_{i}$ with a final derivative results in a linear combination of $D^{2}\left(H^{\dagger} H B_{L} W_{L}\right)$ operators making up a total derivative. Therefore, each $D\left(y_{i}\right)$ equation provides an IBP 
relation among the higher-derivative terms. For example:

$$
\begin{aligned}
D_{\delta \dot{\delta}}\left(y_{1}\right)= & \text { total deriv. }=D_{\delta \dot{\delta}}\left(\left(D H^{\dagger}\right)_{\alpha \dot{\alpha}} H B_{L\{\beta \gamma\}} W_{L\{\xi \eta\}} \epsilon^{\beta x} \epsilon^{\gamma y}\right) \epsilon^{\dot{\alpha} \dot{\delta}} \epsilon^{\alpha \delta} \\
= & \left(\left(D H^{\dagger}\right)_{\alpha \dot{\alpha}}(D H)_{\delta \dot{\delta}} B_{\{\beta \gamma\}} W_{\{\xi \eta\}}+\left(D H^{\dagger}\right)_{\alpha \dot{\alpha}} H\left(D B_{L}\right)_{\{\delta \beta \gamma\}, \dot{\delta}} W_{L\{\xi \eta\}}+\right. \\
& \left.\left(D H^{\dagger}\right)_{\alpha \dot{\alpha}} H B_{L\{\beta \gamma\}}\left(D W_{L}\right)_{\{\delta \xi \eta\}, \dot{\delta}}\right) \epsilon^{\dot{\alpha} \dot{\delta}} \epsilon^{\alpha \delta} \epsilon^{\beta \xi} \epsilon^{\gamma \eta} \\
= & x_{1}+x_{3}+x_{4},
\end{aligned}
$$

where we will usually have to do some index juggling to get the contractions in $D\left(y_{1}\right)$ to match those of the $x_{i}$. Notice that in addition to adding the derivative, we have to specify (and stick to) a convention on how to stitch up the remaining indices. The net result of $D\left(y_{1}\right)$ is that $x_{1}, x_{3}$ and $x_{4}$ are not all independent, i.e. given two we can generate the third.

Following this logic, each of the other $D\left(y_{i}\right)$ provides a relation, or constraint, among the $x_{i}$. If each of the $D\left(y_{i}\right)$ were independent, this would tell us that the true number of independent operators - including IBP relations - is \# $D^{2}$ operators - \#D operators, $\# x_{i}-\# y_{i}$, or more generally (\# operators at $\left.\mathcal{O}\left(D^{m}\right)\right)-\left(\#\right.$ operators at $\mathcal{O}\left(D^{m-1}\right)$ ). However, in practice, the constraint equations are often redundant, making the number of independent constraints $<\# y_{i}$.

To get at the number of independent constraints, we can write the constraint equations as a matrix with each $D\left(y_{i}\right)$ as a row acting on a vector of $x_{i}$, and then determine its rank. For the example here, carrying out the same manipulations as in eq. (2.19) for the rest of the $y_{i}$, we find:

$$
\left(\begin{array}{ccccccc}
1 & 0 & 1 & 1 & 0 & 0 & 0 \\
0 & -1 & -\frac{1}{2} & \frac{1}{2} & 0 & 0 & 0 \\
1 & 0 & 0 & 0 & 1 & 1 & 0 \\
0 & 1 & 0 & 0 & -\frac{1}{2} & \frac{1}{2} & 0 \\
0 & 0 & 1 & 0 & 1 & 0 & 1 \\
0 & 0 & 0 & 1 & 0 & 1 & 1
\end{array}\right)\left(\begin{array}{l}
x_{1} \\
x_{2} \\
x_{3} \\
x_{4} \\
x_{5} \\
x_{6} \\
x_{7}
\end{array}\right)=M_{\mathrm{IBP}} \cdot \vec{x}=0
$$

The constraint matrix $M_{\mathrm{IBP}}$ has rank 5, indicating only five of the six IBP relations are actually independent. Applying five constraints to seven operators leaves us with two independent operators, in agreement with the Hilbert series counting. While tedious, this constraint procedure can be applied to any operator type with derivatives (fermion or bosons), including those with multiple electroweak contractions. After this treatment of IBP relations was put forward in [45] and applied to the SMEFT, later work [4] showed it had missed some operators by overcounting IBP relations. Revisiting the constraint procedure here, we find that the error in ref. [45] did not lie in the method, but was due to mathematical mistakes made when applying the method. ${ }^{12}$ When applied correctly, the number of independent operators (after IBP) found using the constraint method agrees

\footnotetext{
${ }^{12}$ In particular, some of the error in ref. [45] can be traced to a faulty shortcut the authors used to determine when a full matrix/rank treatment of the constraints was necessary, while in other circumstances it was just algebraic error. Clearly this method would benefit from automation, possibly along the lines of ref. [78].
} 
with the Hilbert series counting (at least at dimension $\leq 8$ ). The payoff of the constraint method is that it gives us the actual form of the operators and tells us which operators are related by IBP and which are not. For the example at hand, after row-reducing $M_{\text {IBP }}$ we find it can be distilled to the following relations:

$$
\begin{aligned}
x_{1}-x_{7} & =0, \quad x_{2}+x_{6}+\frac{x_{7}}{2}=0, \quad x_{3}-x_{6}=0, \\
x_{4}+x_{6}+x_{7} & =0, \quad x_{5}+x_{6}+x_{7}=0 .
\end{aligned}
$$

From these relations, we see that $x_{1}$ and $x_{2}$ are sufficient to generate all seven operators. Thus, given a combination $c_{i} x_{i}$, we can IBP repeatedly (throwing away surface terms) and collapse the sum into $c_{1, \mathrm{eff}} x_{1}+c_{2 \text {,eff }} x_{2}$, with $c_{\text {eff }}$ some linear combination of the initial $c_{i}$. We could collapse the sum into other pairs of operators, such as $\left\{x_{1}, x_{3}\right\}$ or $\left\{x_{6}, x_{7}\right\}$, however there are also other pairs, such as $\left\{x_{4}, x_{5}\right\}$, that we could not reduce to. Choosing $\left\{x_{1}, x_{2}\right\}$ to span the set, reintroducing the $\mathrm{SU}(2)_{w}$ indices, and performing steps 4 and 6 from section 2.1, we are left with:

$$
\begin{aligned}
& \mathcal{O}_{2 D H 2 B W 1}= \operatorname{Tr}\left(D_{\mu} H^{\dagger} \tau^{I} D^{\mu} H\right)\left(B_{L}^{\rho \sigma} W_{L, \rho \sigma}^{I}-i B_{L}^{\rho \sigma} \tilde{W}_{L, \rho \sigma}^{I}\right) \\
& \mathcal{O}_{2 D H 2 B W 2}=\operatorname{Tr}\left(D_{\mu} H^{\dagger} \tau^{I} D_{\nu} H\right)\left(B_{L}^{\mu \kappa} W_{L, \kappa}^{I \nu}-B_{L}^{\nu \kappa} W_{L, \kappa}^{I \mu}+\frac{i}{2} B_{L}^{\nu \kappa} \tilde{W}_{L, \kappa}^{\mu}-\frac{i}{2} B_{L}^{\mu \kappa} \tilde{W}_{L, \kappa}^{\nu}\right. \\
&\left.+\frac{i}{2} \tilde{B}_{L}^{\nu \kappa} W_{L, \kappa}^{\mu}-\frac{i}{2} \tilde{B}_{L}^{\mu \kappa} W_{L, \kappa}^{\nu}\right)
\end{aligned}
$$

where, as in eq. (2.14), we have converted to $H_{\frac{2}{2}}^{\dagger}$ format.

One remaining question is the origin of the IBP relation redundancies. Combining several of the $y_{i}$ and manipulating indices, we see that some combinations of the constraint operators can be expressed as a total derivative:

$$
y_{2}+y_{4}+\frac{1}{2}\left(y_{5}-y_{6}\right)=D_{\alpha \dot{\alpha}}\left(H^{\dagger} H B_{\{\beta \gamma\}} W_{\{\xi \eta\}} \frac{1}{2} \epsilon^{\gamma \xi}\left(\epsilon^{\alpha \beta}+\epsilon^{\alpha \eta}\right)\right)
$$

When we apply a final derivative, eq. (2.22) connects the constraints from $D\left(y_{2}\right), D\left(y_{4}\right)$, $D\left(y_{5}\right)$, and $D\left(y_{6}\right)$ so they are no longer independent. For the case here, we have a single relation among the $y_{i}$, so the number of independent constraints is reduced by one, from six to five. To better understand why this occurs, notice that the operator in the parentheses of eq. (2.22) transforms as a $(0,1)$ Lorentz representation. Applying $D^{2}$ to this combination will always give zero, since $D^{2}$ has Lorentz irreducible representations $(0,0) \oplus(1,1)$, which cannot form a singlet with $(0,1)$. Enforcing this fact - that $D^{2} \otimes(0,1)=0$ - results in relations among the $\mathcal{O}(D)$ operators. ${ }^{13}$

\footnotetext{
${ }^{13}$ Technically, we are only interested in $(0,1)$ or $(1,0)$ operators that are not themselves a total derivative. The Hilbert series iteratively removes total derivative terms, as explained in [4]. In the constraint method shown here, $D^{m-2}, D^{m-3} \ldots$ total derivatives all show up as relations among rows of $M_{\mathrm{IBP}}$.
} 


\begin{tabular}{|c|c|c|c|}
\hline $\mathcal{O}_{8, H}$ & $\left(H^{\dagger} H\right)^{4}$ & $\mathcal{O}_{8, W}$ & $\epsilon_{I J K}\left(H^{\dagger} H\right) W^{\mu \nu, I} W_{\nu \rho}^{J} W_{\mu}^{\rho, K}$ \\
$\mathcal{O}_{8, H B}$ & $\left(H^{\dagger} H\right)^{2} B_{\mu \nu} B^{\mu \nu}$ & $\mathcal{O}_{8, \tilde{W}}$ & $\epsilon_{I J K}\left(H^{\dagger} H\right) W^{\mu \nu, I} \widetilde{W}_{\nu \rho}^{J} W_{\mu}^{\rho, K}$ \\
$\mathcal{O}_{8, H \tilde{B}}$ & $\left(H^{\dagger} H\right)^{2} B_{\mu \nu} \widetilde{B}^{\mu \nu}$ & $\mathcal{O}_{8, H G}$ & $\delta_{A B}\left(H^{\dagger} H\right)^{2} G_{\mu \nu}^{A} G^{\mu \nu, B}$ \\
$\mathcal{O}_{8, H W B}$ & $\delta_{I J}\left(H^{\dagger} H\right)\left(H^{\dagger} \tau^{I} H\right) B_{\mu \nu} W^{\mu \nu, J}$ & $\mathcal{O}_{8, H \tilde{G}}$ & $\delta_{A B}\left(H^{\dagger} H\right)^{2} G_{\mu \nu}^{A} \widetilde{G}^{\mu \nu, B}$ \\
$\mathcal{O}_{8, H \tilde{W} B}$ & $\delta_{I J}\left(H^{\dagger} H\right)\left(H^{\dagger} \tau^{I} H\right) B_{\mu \nu} \widetilde{W}^{\mu \nu, J}$ & $\mathcal{O}_{8, G}$ & $f_{A B C}\left(H^{\dagger} H\right) G^{\mu \nu, A} G_{\nu \rho}^{B} G_{\mu}^{\rho, C}$ \\
$\mathcal{O}_{8, H W}$ & $\delta_{I J}\left(H^{\dagger} H\right)^{2} W_{\mu \nu}^{I} W^{\mu \nu, J}$ & $\mathcal{O}_{8, \tilde{G}}$ & $f_{A B C}\left(H^{\dagger} H\right) G^{\mu \nu, A} \widetilde{G}_{\nu \rho}^{B} G_{\mu}^{\rho, C}$ \\
$\mathcal{O}_{8, H \tilde{W}}$ & $\delta_{I J}\left(H^{\dagger} H\right)^{2} W_{\mu \nu}^{I} \widetilde{W}^{\mu \nu, J}$ & & \\
$\mathcal{O}_{8, H W 2}$ & $\delta_{I K} \delta_{J M}\left(H^{\dagger} \tau^{I} H\right)\left(H^{\dagger} \tau^{J} H\right) W_{\mu \nu}^{K} W^{\mu \nu, M}$ & & \\
$\mathcal{O}_{8, H \tilde{W} 2}$ & $\delta_{I K} \delta_{J M}\left(H^{\dagger} \tau^{I} H\right)\left(H^{\dagger} \tau^{J} H\right) W_{\mu \nu}^{K} \widetilde{W}^{\mu \nu, M}$ & & \\
$\mathcal{O}_{8, H W B 2}$ & $\epsilon_{I J K}\left(H^{\dagger} \tau^{I} H\right) B_{\mu}^{\nu} W_{\nu \rho}^{J} W^{\mu \rho, K}$ & & \\
$\mathcal{O}_{8, H W \tilde{B} 2}$ & $\epsilon_{I J K}\left(H^{\dagger} \tau^{I} H\right)\left(\widetilde{B}^{\mu \nu} W_{\nu \rho}^{J} W_{\mu}^{\rho, K}+B^{\mu \nu} W_{\nu \rho}^{J} \widetilde{W}_{\mu}^{\rho, K}\right)$ & \multicolumn{2}{|l}{} \\
\cline { 1 - 1 }
\end{tabular}

Table 4. The 17 derivative-free operators after conversion to the standard $X, \widetilde{X}$ notation for the field-strength tensors and with $H^{\dagger}$ in the $\overline{2}$ representation.

\section{Dimension-8 operators relevant for $p p \rightarrow h W$}

Having worked through a few examples, we now present the results of the operator extraction for the full set of terms listed in eqs. (2.1)-(2.4).

- For the bosonic terms there are a total of 17 operators with no derivatives, corresponding to the eight terms in eq. (2.1) and their hermitian conjugates, along with the single self-hermitian term. The set is listed in table 4. As explained in section 2.1, we have made some choices about how to display indices (e.g. triplets vs. doublets) and what linear combinations to take to form operators with simple properties under CP transformations. These choices constitute a choice of basis.

- Table 5 contains the bosonic operators with two derivatives. There are 26 operators, corresponding to ten terms in eq. (2.2) plus their hermitian conjugates, and the six self-hermitian terms. Because there are derivatives, there is even more choice than in table 4. When possible, we have opted to put the derivatives on the Higgs fields as this makes implementing the operators into FeynRules easier.

- Table 6 contains the three bosonic operators at $\mathcal{O}\left(D^{4}\right)$. When forming these operators (and all others in this section) we have ignored overall signs or numerical factors.

- Finally, table 7 contains the $N_{f}=1$ fermionic operators that contribute to $p p \rightarrow h W$, either by contributing to the $\bar{q} q W$ vertices or through direct four-point contact terms. There are no terms with even numbers of derivatives, as operators of that sort always contain a mixed chirality fermion pair and therefore do not interfere with SM $p p \rightarrow h W$ amplitudes. Here, $H^{\dagger} \overleftrightarrow{D}^{\mu} H=\left(D^{\mu} H^{\dagger}\right) H-H^{\dagger}\left(D^{\mu} H\right)$ 


\begin{tabular}{|c|c|c|c|}
\hline $\mathcal{O}_{8, H D}$ & $\left(H^{\dagger} H\right)^{2}\left(D_{\mu} H^{\dagger} D^{\mu} H\right)$ & $\mathcal{O}_{8, D H \tilde{W} 3 b}$ & $\epsilon_{I J K}\left(D^{\mu} H^{\dagger} \tau^{I} D^{\nu} H\right)\left(W_{\mu \rho}^{J} \widetilde{W}_{\nu}^{\rho, K}+\widetilde{W}_{\mu \rho}^{J} W_{\nu}^{\rho, K}\right)$ \\
\hline $\mathcal{O}_{8, H D 2}$ & $\delta_{I J}\left(H^{\dagger} H\right)\left(H^{\dagger} \tau^{I} H\right)\left(D^{\mu} H^{\dagger} \tau^{J} D_{\mu} H\right)$ & $\mathcal{O}_{8, D H W B}$ & $\delta_{I J}\left(D^{\mu} H^{\dagger} \tau^{I} D_{\mu} H\right) B^{\rho \sigma} W_{\rho \sigma}^{J}$ \\
\hline $\mathcal{O}_{8, D H B}$ & $\left(D^{\mu} H^{\dagger} D^{\nu} H\right) B_{\mu \rho} B_{\nu}^{\rho}$ & $\mathcal{O}_{8, D H \tilde{W} B}$ & $\delta_{I J}\left(D^{\mu} H^{\dagger} \tau^{I} D_{\mu} H\right) B^{\rho \sigma} \widetilde{W}_{\rho \sigma}^{J}$ \\
\hline $\mathcal{O}_{8, D H B 2}$ & $\left(D^{\mu} H^{\dagger} D_{\mu} H\right) B^{\rho \sigma} B_{\rho \sigma}$ & $\mathcal{O}_{8, D H W B 2}$ & $i \delta_{I J}\left(D^{\mu} H^{\dagger} \tau^{I} D^{\nu} H\right)\left(B_{\mu \rho} W_{\nu}^{\rho, J}-B_{\nu \rho} W_{\mu}^{\rho, J}\right)$ \\
\hline $\mathcal{O}_{8, D H \tilde{B} 2}$ & $\left(D^{\mu} H^{\dagger} D_{\mu} H\right) B^{\rho \sigma} \widetilde{B}_{\rho \sigma}$ & $\mathcal{O}_{8, D H W B 3}$ & $\delta_{I J}\left(D^{\mu} H^{\dagger} \tau^{I} D^{\nu} H\right)\left(B_{\mu \rho} W_{\nu}^{\rho, J}+B_{\nu \rho} W_{\mu}^{\rho, J}\right)$ \\
\hline $\mathcal{O}_{8, D H G}$ & $\delta_{A B}\left(D^{\mu} H^{\dagger} D^{\nu} H\right) G_{\mu \rho}^{A} G_{\nu}^{\rho, B}$ & $\mathcal{O}_{8, D H \tilde{W} B 2}$ & $\delta_{I J}\left(D^{\mu} H^{\dagger} \tau^{I} D^{\nu} H\right)\left(B_{[\mu}^{\rho} \widetilde{W}_{\nu] \rho}^{J}-\widetilde{B}_{[\mu}^{\rho} W_{\nu] \rho}^{J}\right)$ \\
\hline $\mathcal{O}_{8, D H G 2}$ & $\delta_{A B}\left(D^{\mu} H^{\dagger} D_{\mu} H\right) G^{\rho \sigma, A} G_{\rho \sigma}^{B}$ & $\mathcal{O}_{8, D H \tilde{W} B 3}$ & $\delta_{I J}\left(D^{\mu} H^{\dagger} \tau^{I} D^{\nu} H\right)\left(B_{\{\mu}^{\rho} \widetilde{W}_{\nu\} \rho}^{J}+\widetilde{B}_{\{\mu}^{\rho} W_{\nu\} \rho}^{J}\right)$ \\
\hline $\mathcal{O}_{8, D H \tilde{G} 2}$ & $\delta_{A B}\left(D^{\mu} H^{\dagger} D_{\mu} H\right) G^{\rho \sigma, A} \widetilde{G}_{\rho \sigma}^{B}$ & $\mathcal{O}_{8, H D H B}$ & $i\left(H^{\dagger} H\right)\left(D_{\mu} H^{\dagger} D_{\nu} H\right) B^{\mu \nu}$ \\
\hline $\mathcal{O}_{8, D H W}$ & $\delta_{I J}\left(D^{\mu} H^{\dagger} D^{\nu} H\right) W_{\mu \rho}^{I} W_{\nu}^{\rho, J}$ & $\mathcal{O}_{8, H D H \tilde{B}}$ & $i\left(H^{\dagger} H\right)\left(D_{\mu} H^{\dagger} D_{\nu} H\right) \widetilde{B}^{\mu \nu}$ \\
\hline $\mathcal{O}_{8, D H W 2}$ & $\delta_{I J}\left(D^{\mu} H^{\dagger} D_{\mu} H\right) W^{\rho \sigma, I} W_{\rho \sigma}^{J}$ & $\mathcal{O}_{8, H D H W}$ & $i \delta_{I J}\left(H^{\dagger} H\right)\left(D^{\mu} H^{\dagger} \tau^{I} D^{\nu} H\right) W_{\mu \nu}^{J}$ \\
\hline $\mathcal{O}_{8, D H \tilde{W} 2}$ & $\delta_{I J}\left(D^{\mu} H^{\dagger} D_{\mu} H\right) W^{\rho \sigma, I} \widetilde{W}_{\rho \sigma}^{J}$ & $\mathcal{O}_{8, H D H \tilde{W}}$ & $i \delta_{I J}\left(H^{\dagger} H\right)\left(D^{\mu} H^{\dagger} \tau^{I} D^{\nu} H\right) \widetilde{W}_{\mu \nu}^{J}$ \\
\hline $\mathcal{O}_{8, D H W 3}$ & $\epsilon_{I J K}\left(D^{\mu} H^{\dagger} \tau^{I} D^{\nu} H\right) W_{\mu \rho}^{J} W_{\nu}^{\rho, K}$ & $\mathcal{O}_{8, H D H W 2}$ & $i \epsilon_{I J K}\left(H^{\dagger} \tau^{I} H\right)\left(D^{\mu} H^{\dagger} \tau^{J} D^{\nu} H\right) W_{\mu \nu}^{K}$ \\
\hline $\mathcal{O}_{8, D H \tilde{W} 3 a}$ & $\epsilon_{I J K}\left(D^{\mu} H^{\dagger} \tau^{I} D^{\nu} H\right)\left(W_{\mu \rho}^{J} \widetilde{W}_{\nu}^{\rho, K}-\widetilde{W}_{\mu \rho}^{J} W_{\nu}^{\rho, K}\right)$ & $\mathcal{O}_{8, H D H \tilde{W} 2}$ & $i \epsilon_{I J K}\left(H^{\dagger} \tau^{I} H\right)\left(D^{\mu} H^{\dagger} \tau^{J} D^{\nu} H\right) \widetilde{W}_{\mu \nu}^{K}$ \\
\hline
\end{tabular}

Table 5. The 26 two-derivative operators after conversion to the standard $X, \tilde{X}$ notation (plus linear combinations). Factors of $i$ are included where necessary so that the operators are explicitly self-hermitian with real coefficients.

\begin{tabular}{|c|c|}
\hline $\mathcal{O}_{8,4 D 1}$ & $\left(D_{\mu} H^{\dagger} D_{\nu} H\right)\left(D^{\nu} H^{\dagger} D^{\mu} H\right)$ \\
$\mathcal{O}_{8,4 D 2}$ & $\left(D_{\mu} H^{\dagger} D_{\nu} H\right)\left(D^{\mu} H^{\dagger} D^{\nu} H\right)$ \\
$\mathcal{O}_{8,4 D 3}$ & $\left(D^{\mu} H^{\dagger} D_{\mu} H\right)\left(D^{\nu} H^{\dagger} D_{\nu} H\right)$ \\
\hline
\end{tabular}

Table 6. The explicit forms of the three bosonic dimension- 8 operators containing at least one Higgs field and four derivatives. These three operators have three independent real coefficients.

The left-hand column of table 7 shows the 12 operators derived from $D\left(Q^{\dagger} Q H^{\dagger} H W_{L, R}\right)$, grouped into $\mathrm{CP}$-even/odd pairs. These operators are each accompanied by a real coefficient in the Lagrangian, however one could also combine each pair, e.g. $\mathcal{O}_{8, Q W 1}$ and $\mathcal{O}_{8, Q \tilde{W} 1}$, into a complex operator with complex coefficient. The 8 operators on the right-hand side correspond to $D\left(Q^{\dagger} Q H^{2}\left(H^{\dagger}\right)^{2}\right)$ and $D^{3}\left(Q^{\dagger} Q H^{\dagger} H\right)$. These operators are accompanied by real coefficients. Throughout this list we have chosen to put derivatives on the Higgs fields whenever possible. However, while it is possible to form an invariant with three derivatives on Higgs fields, $Q^{\dagger} Q D^{2} H^{\dagger} D H$ and $Q^{\dagger} Q D H^{\dagger} D^{2} H$ (each with two electroweak index contraction possibilities), the two are not independent under IBP so we cannot span the full set of $D^{3}\left(Q^{\dagger} Q H^{\dagger} H\right)$ operators with them. Rather than choose one, $Q^{\dagger} Q D^{2} H^{\dagger} D H$ or $Q^{\dagger} Q D H^{\dagger} D^{2} H$, we have opted for a more symmetric choice involving two derivatives on Higgs fields and one on a fermion field.

Using table 7 one can easily write down similar operators involving right-handed fermions. For left-handed leptons we just need to replace $Q \rightarrow L$ since the $\mathrm{SU}(3)_{c}$ structure played no role; similarly, trading in $Q$ for $u_{c}, d_{c}$ or $e_{c}$, only the $\mathrm{SU}(2)_{w}$-singlet fermion combinations $\mathcal{O}_{8, Q 1}, \mathcal{O}_{8,3 Q 1}, \mathcal{O}_{8,3 Q 3}$ and $\mathcal{O}_{8, Q W 1}, \mathcal{O}_{8, Q \tilde{W} 1}, \mathcal{O}_{8, Q W 2}, \mathcal{O}_{8, Q \tilde{W} 2}$ are allowed. 


\begin{tabular}{|c|c|c|c|}
\hline $\mathcal{O}_{8, Q W 1}$ & $\delta_{I J}\left(Q^{\dagger} \bar{\sigma}^{\nu} Q\right) D^{\mu}\left(H^{\dagger} \tau^{I} H\right) W_{\mu \nu}^{J}$ & $\mathcal{O}_{8, Q 1}$ & $i\left(Q^{\dagger} \bar{\sigma}^{\mu} Q\right)\left(H^{\dagger} \overleftrightarrow{D}^{\mu} H\right)\left(H^{\dagger} H\right)$ \\
\hline $\mathcal{O}_{8, Q \tilde{W} 1}$ & $\delta_{I J}\left(Q^{\dagger} \bar{\sigma}^{\nu} Q\right) D^{\mu}\left(H^{\dagger} \tau^{I} H\right) \tilde{W}_{\mu \nu}^{J}$ & $\mathcal{O}_{8, Q 2}$ & $\begin{array}{c}i \delta_{I J}\left(Q^{\dagger} \bar{\sigma}^{\mu} \tau^{I} Q\right)\left(\left(\overleftrightarrow{D}_{\mu} H^{\dagger} \tau^{J} H\right)\left(H^{\dagger} H\right)+\right. \\
\left.\left(\overleftrightarrow{D}_{\mu} H^{\dagger} H\right)\left(H^{\dagger} \tau^{J} H\right)\right)\end{array}$ \\
\hline $\mathcal{O}_{8, Q W 2}$ & $i \delta_{I J}\left(Q^{\dagger} \bar{\sigma}^{\nu} Q\right)\left(H^{\dagger} \overleftrightarrow{D^{\mu}} \tau^{I} H\right) W_{\mu \nu}^{J}$ & $\mathcal{O}_{8, Q 3}$ & $i \epsilon_{I J K}\left(Q^{\dagger} \bar{\sigma}^{\mu} \tau^{I} Q\right)\left(H^{\dagger} \overleftrightarrow{D^{\mu}} \tau^{J} H\right)\left(H^{\dagger} \tau^{K} H\right)$ \\
\hline $\mathcal{O}_{8, Q \tilde{W} 2}$ & $i \delta_{I J}\left(Q^{\dagger} \bar{\sigma}^{\nu} Q\right)\left(H^{\dagger} \overleftrightarrow{D}^{\mu} \tau^{I} H\right) \tilde{W}_{\mu \nu}^{J}$ & $\mathcal{O}_{8, Q 4}$ & $\epsilon_{I J K}\left(Q^{\dagger} \bar{\sigma}^{\mu} \tau^{I} Q\right)\left(H^{\dagger} \tau^{J} H\right) D_{\mu}\left(H^{\dagger} \tau^{K} H\right)$ \\
\hline $\mathcal{O}_{8, Q W 3}$ & $\delta_{I J}\left(Q^{\dagger} \bar{\sigma}^{\nu} \tau^{I} Q\right) D^{\mu}\left(H^{\dagger} H\right) W_{\mu \nu}^{J}$ & $\mathcal{O}_{8,3 Q 1}$ & $i\left(Q^{\dagger} \bar{\sigma}^{\mu} D^{\nu} Q\right)\left(D_{(\mu \nu)}^{2} H^{\dagger} H\right)+h . c$. \\
\hline $\mathcal{O}_{8, Q \tilde{W} 3}$ & $\delta_{I J}\left(Q^{\dagger} \bar{\sigma}^{\nu} \tau^{I} Q\right) D^{\mu}\left(H^{\dagger} H\right) \tilde{W}_{\mu \nu}^{J}$ & $\mathcal{O}_{8,3 Q 2}$ & $i \delta_{I J}\left(Q^{\dagger} \bar{\sigma}^{\mu} \tau^{I} D^{\nu} Q\right)\left(D_{(\mu \nu)}^{2} H^{\dagger} \tau^{J} H\right)+$ h.c. \\
\hline $\mathcal{O}_{8, Q W 4}$ & $i \delta_{I J}\left(Q^{\dagger} \bar{\sigma}^{\nu} \tau^{I} Q\right)\left(H^{\dagger} \overleftrightarrow{D}^{\mu} H\right) W_{\mu \nu}^{J}$ & $\mathcal{O}_{8,3 Q 3}$ & $i\left(Q^{\dagger} \bar{\sigma}^{\mu} D^{\nu} Q\right)\left(H^{\dagger} D_{(\mu \nu)}^{2} H\right)+$ h.c. \\
\hline $\mathcal{O}_{8, Q \tilde{W} 4}$ & $i \delta_{I J}\left(Q^{\dagger} \bar{\sigma}^{\nu} \tau^{I} Q\right)\left(H^{\dagger} \overleftrightarrow{D}^{\mu} H\right) \tilde{W}_{\mu \nu}^{J}$ & $\mathcal{O}_{8,3 Q 4}$ & $i \delta_{I J}\left(Q^{\dagger} \bar{\sigma}^{\mu} \tau^{I} D^{\nu} Q\right)\left(H^{\dagger} \tau^{J} D_{(\mu \nu)}^{2} H\right)+$ h.c. \\
\hline $\mathcal{O}_{8, Q W 5}$ & $\epsilon_{A B C}\left(Q^{\dagger} \bar{\sigma}^{\nu} \tau^{A} Q\right) D^{\mu}\left(H^{\dagger} \tau^{B} H\right) W_{\mu \nu}^{C}$ & & \\
\hline $\mathcal{O}_{8, Q \tilde{W} 5}$ & $\epsilon_{A B C}\left(Q^{\dagger} \bar{\sigma}^{\nu} \tau^{A} Q\right) D^{\mu}\left(H^{\dagger} \tau^{B} H\right) \tilde{W}_{\mu \nu}^{C}$ & & \\
\hline $\mathcal{O}_{8, Q W 6}$ & $i \epsilon_{A B C}\left(Q^{\dagger} \bar{\sigma}^{\nu} \tau^{A} Q\right)\left(H^{\dagger} \overleftrightarrow{D}^{\mu} \tau^{B} H\right) W_{\mu \nu}^{C}$ & & \\
\hline $\mathcal{O}_{8, Q \tilde{W} 6}$ & $i \epsilon_{A B C}\left(Q^{\dagger} \bar{\sigma}^{\nu} \tau^{A} Q\right)\left(H^{\dagger} \overleftrightarrow{D}^{\mu} \tau^{B} H\right) \tilde{W}_{\mu \nu}^{C}$ & & \\
\hline
\end{tabular}

Table 7. The $20\left(N_{f}=1\right)$ operators involving quark, $W$-boson and Higgs fields that are relevant for the phenomenological study of $p p \rightarrow h W^{ \pm}$.

\section{Application: $p p \rightarrow h W^{ \pm}$}

To investigate the effect of dimension- 8 operators we focus on one process: Higgs boson production in association with a $W$ boson at the LHC. See refs. [79-84] for relevant experimental results.

Combining the operators in tables $4-7$ with dimension- 6 operators (enumerated in appendix B), higher-dimensional terms manifest in a number of ways. Bosonic operators directly enter into $p p \rightarrow h W^{ \pm}$by modifying the $h W W$ vertex, while fermionic operators (e.g. table 7 ) either modify the $\bar{q} q W^{ \pm}$vertex or enter as $\bar{q} q h W^{ \pm}$contact terms. Additionally, higher-dimensional operators introduce corrections to the SM field kinetic terms. For instance, $\mathcal{O}_{8, H B}$ leads to a correction to the $\mathrm{U}(1)$ kinetic term, $c_{8, H B}\left(v^{4} / \Lambda^{4}\right) B_{\mu \nu} B^{\mu \nu}$, and similarly $\mathcal{O}_{8, H D}$ leads to a correction to the Higgs kinetic term. To ensure that all fields are canonically normalized we must make a set of field redefinitions. These redefinitions lead to shifts (relative to the SM) in how couplings and masses in the Lagrangian are related to experimental inputs. The procedure to relate experimental inputs and electroweak parameters in the presence of dimension- 6 operators has been worked out in refs. [85, 86]. Dimension- 8 effects can be handled by the same method, though they introduce a dependence of the electroweak parameters at order $1 / \Lambda^{4}$ and need to be handled with care. Furthermore, our restriction to $N_{f}=1$ operators impacts the electroweak parameter shifts. Specifically, this restricstion removes four-fermion contributions to $G_{F}$, as $G_{F}$ is taken from muon decay and necessarily involves communication among different generations. Removing the four-fermion contributions, the only higher dimensional operators that affect the 
interpretation of $G_{F}$ in terms of theory inputs are operators that shift the coupling of leptons to the $W$ boson. Details of the electroweak input procedure, including the $G_{F}$ subtleties, are presented in appendix D.

After carrying out the normalization and EW input procedure, we next sketch the Feynman rules for the $\bar{q} q W, h W W$, and $\bar{q} q h W$ vertices. We take all momenta to be ingoing and enforce on-shell conditions on the Higgs and fermion fields, but not on the gauge bosons. While it is possible to remove the dependence on one field momentum in each vertex by imposing momentum conservation, we choose not to do so. To be more compact and make the different Lorentz structures clearer, we first express the Feynman rules in terms of form factors $c_{f f V i}, c_{h W W i}, c_{f f W h i}:^{14}$

$d\left(p_{2}\right)$

$\sim W_{\mu}^{+}\left(p_{3}\right)=\bar{v}\left(p_{2}\right) \gamma^{\mu}\left(c_{q q V 0}+c_{q q V 1} \frac{p_{3}^{2}}{2}\right) P_{L} u\left(p_{1}\right)$

$u\left(p_{1}\right)$

$$
\begin{aligned}
& W_{\mu}^{+}\left(p_{2}\right) \\
& h\left(p_{1}\right)--\left\{\begin{array}{r}
=\left(c_{h V V 0} \eta^{\mu \nu}+c_{h V V 1}\left(\left(p_{2} \cdot p_{3}\right) \eta^{\mu \nu}-p_{2}^{\nu} p_{3}^{\mu}\right)\right. \\
+c_{h V V 2}\left(\left(p_{1} \cdot p_{3}\right) \eta^{\mu \nu}-p_{1}^{\nu} p_{3}^{\mu}\right)
\end{array}\right. \\
& \left.W_{\nu}^{-}\left(p_{3}\right) \quad-c_{h V V 2}^{*}\left(\left(p_{1} \cdot p_{2}\right) \eta^{\mu \nu}-p_{1}^{\mu} p_{2}^{\nu}\right)\right)
\end{aligned}
$$

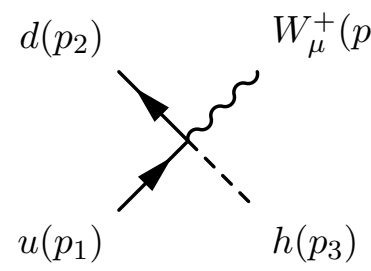

$$
\begin{aligned}
=\bar{v}\left(p_{2}\right)\left(\gamma^{\mu}(\right. & c_{q q W h 0}+c_{q q W h 2}\left(p_{3} \cdot p_{4}\right) \\
& +c_{q q W h 3}\left(p_{1} \cdot\left(p_{3}+p_{4}\right)\right)+c_{q q W h 4}\left(p_{2} \cdot\left(p_{3}+p_{4}\right)\right) \\
& +\not p_{3}\left(c_{q q W h 1} p_{3}^{\mu}+c_{q q W h 3} p_{1}^{\mu}+c_{q q W h 4} p_{2}^{\mu}\right) \\
& \left.\left.+\not p_{4}\left(-c_{q q W h 2} p_{3}^{\mu}+c_{q q W h 3} p_{1}^{\mu}+c_{q q W h 4} p_{2}^{\mu}\right)\right)\right) P_{L} u\left(p_{2}\right)
\end{aligned}
$$

The full expressions for the form factors are provided in appendix E. ${ }^{15}$

Using these vertices to calculate $\hat{\sigma}\left(p p \rightarrow h W^{+}\right)$in terms of the dimension- 6 or -8 coefficients, the full expression is not particularly illuminating. However, a quick way to see how dimension- 8 effects enter and what are the most important operators is to take the limit of large $\hat{s}$, as that will expose differences in the high energy behavior of dimension- 6 vs. dimension-8. We find, after plugging in the full expressions for the form factors:

$$
\begin{array}{r}
\hat{\sigma}\left(p p \rightarrow W^{+} h\right) \sim\left(\frac{\hat{e}^{2}}{4608 \pi \sin ^{4} \hat{\theta}}\right) \frac{\hat{v}^{2}}{m_{W}^{2}} \frac{\hat{s}}{\Lambda^{4}}\left(e^{2}\left(c_{8,3 Q 1}-c_{8,3 Q 2}+c_{8,3 Q 3}+c_{8,3 Q 4}\right)\right. \\
\left.+8 \sin ^{2} \theta\left(c_{H q}^{(3)}\right)^{2}\right)+\mathcal{O}\left(\hat{s}^{0}\right) .
\end{array}
$$

\footnotetext{
${ }^{14}$ For simplicity, we neglect CP-odd operators throughout this discussion.

${ }^{15}$ While we have presented off-shell vertices, it would be interesting to explore these results using on-shell amplitude techniques along the lines of ref. [43].
} 
The largest growth is linear in $\hat{s}$, as expected from simple power-counting arguments, and the operators that enter are a dimension- 6 contact term (squared), and a combination of $D^{3}\left(Q^{\dagger} Q H^{\dagger} H\right)$ dimension-8 terms. The fact that $D^{3}\left(Q^{\dagger} Q H^{\dagger} H\right)$ terms are the only dimension-8 terms to appear is not surprising. Fermionic operators with fewer derivatives contain additional Higgs fields and can only contribute to $p p \rightarrow h W^{+}$if multiple Higgs fields are set to their vevs (more vevs in the amplitude lead to weaker energy dependence). The only dimension-6 term that contributes in eq. (4.4) is $\mathcal{O}_{H q}^{(3)}=i\left(Q^{\dagger} \bar{\sigma}^{\mu} \tau^{I} Q\right)\left(\overleftrightarrow{D} H^{\dagger} \tau^{I} H\right)$. However, this operator also modifies fermion couplings to $W$ and $Z$ bosons (through the form factor $c_{f f V 0}$ in eq. (4.1)). Strong constraints on deviations of $W$ and $Z$ couplings to fermions implies that these operators must have small coefficients, see e.g. ref. [8]. Examining eq. (4.1), we see that the $D^{3}\left(Q^{\dagger} Q H^{\dagger} H\right)$ contact terms are not tied to $\bar{q} q V$ modifications. Thus, if we take $c_{H q}^{(3)} \rightarrow 0$ to avoid $\bar{q} q V$ constraints, the part of $\hat{\sigma}(p p \rightarrow$ $h W^{+}$) that grows with energy is controlled by dimension- 8 operators alone. In this case, the cross section contributions from $\left|A_{\text {dim- } 6}\right|^{2}$ are $\propto \frac{v^{4}}{\Lambda^{4}}$, while those from $A_{\mathrm{SM}} \times A_{\text {dim- } 8}$ are $\propto \frac{v^{2} \hat{s}}{\Lambda^{4}}-$ so there are energy regimes where dimension- 8 effects are dominant at $1 / \Lambda^{4}$. To quantitatively evaluate the effects we can expect at the proton level, we turn to numerics.

As a rough estimate of the impact of dimension- 8 operators, we study the rate of $p p \rightarrow h W^{+}$at the LHC $(\sqrt{s}=13 \mathrm{TeV})$ in a scenario with a single dimension-6 operator and all dimension- 8 operators. We choose $\mathcal{O}_{H W}$ as the representative dimension-6 operator and for simplicity define $c_{H W} \equiv 1 / \Lambda_{6}^{2}$ and set all other dimension- 6 coefficients to zero. We take all dimension- 8 operator coefficients to have the same magnitude, $\left|c_{8, i}\right| \equiv 1 / \Lambda_{8}^{4}$, but leave the signs to float since there can be cancellations among different operators (see eq. (4.4)). ${ }^{16}$ For a fixed $c_{H W}\left(\Lambda_{6}\right)$, the limit $\Lambda_{8} \rightarrow \infty$ corresponds to no dimension- 8 effects. Decreasing $\Lambda_{8}$, we add in the dimension- 8 effects. For each $\Lambda_{6}, \Lambda_{8}$, and sign choice for the coefficients, ${ }^{17}$ we fold the parton-level results with parton distribution functions for a $13 \mathrm{TeV}$ LHC, ${ }^{18}$ then calculate the shift in the $p p \rightarrow h W^{+}$rate relative to the SM, $\left|\Delta \mu\left(p p \rightarrow h W^{+}\right)\right|=\left|\left(\sigma\left(p p \rightarrow h W^{+}\right)_{\Lambda_{6}, \Lambda_{8}}-\sigma\left(p p \rightarrow h W^{+}\right)_{S M}\right) / \sigma\left(p p \rightarrow h W^{+}\right)_{S M}\right|$. If we pick the signs of all dimension- 8 coefficients to be positive, the result is shown in the top panels figure 2. In the bottom panels of figure 2 we show the result if we instead choose signs of the dimension- 8 coefficients that enhance their contributions at large $\sqrt{s} .{ }^{19}$

\footnotetext{
${ }^{16}$ If we were to start with a UV theory and derive the SMEFT by integrating out heavy states, the coefficients in front of the higher dimensional operators would be combinations of heavy particle masses and couplings (and potentially loop factors). Rather than deal with these different components, we have absorbed everything into the overall scales $\Lambda_{6}$ and $\Lambda_{8}$. While convenient for us, this convention does obscure the connection between the operator coefficient and the actual mass scale of new physics. For example, $\Lambda_{6}$ may be large either if the new physics scale is high or if the new physics scale is low but maps to a small coupling coefficient of the effective operator.

${ }^{17}$ As demonstrated in refs. [87, 88], it is possible that analyticity and unitarity requirements forbid certain signs for higher dimensional operator coefficients. We ignore this possibility here and assume the coefficients can have either sign.

${ }^{18}$ We use MSTW2008nnlo [89] parton distribution functions with factorization scale set to $\sqrt{\hat{s}}$.

${ }^{19}$ The sign assignment is the following: $c_{8, H D 2}, c_{8, H D H W}, c_{8, Q W 3}, c_{8, Q W 5}, c_{8,3 Q 1}, c_{8,3 Q 3}, c_{8,3 Q 4}$ positive $\left(=+\frac{1}{\Lambda_{8}^{4}}\right)$; and $c_{8, H D}, c_{8, H W B}, c_{8, H W}, c_{8, H W 2}, c_{8, Q 2}, c_{8, Q 3}, c_{8, Q 4}, c_{8, H D H W 2}, c_{8,3 Q 2}$ negative. While dimension- 8 effects are larger for this choice, there is still some cancellation among terms, namely between $c_{8,3 Q 1}, c_{8,3 Q 3}, c_{8,3 Q 4}$ and $c_{8, Q W 3}, c_{8, Q W 5}$. This sign choice should be thought of as a representative example and not the sign choice that maximizes dimension- 8 effects.
} 

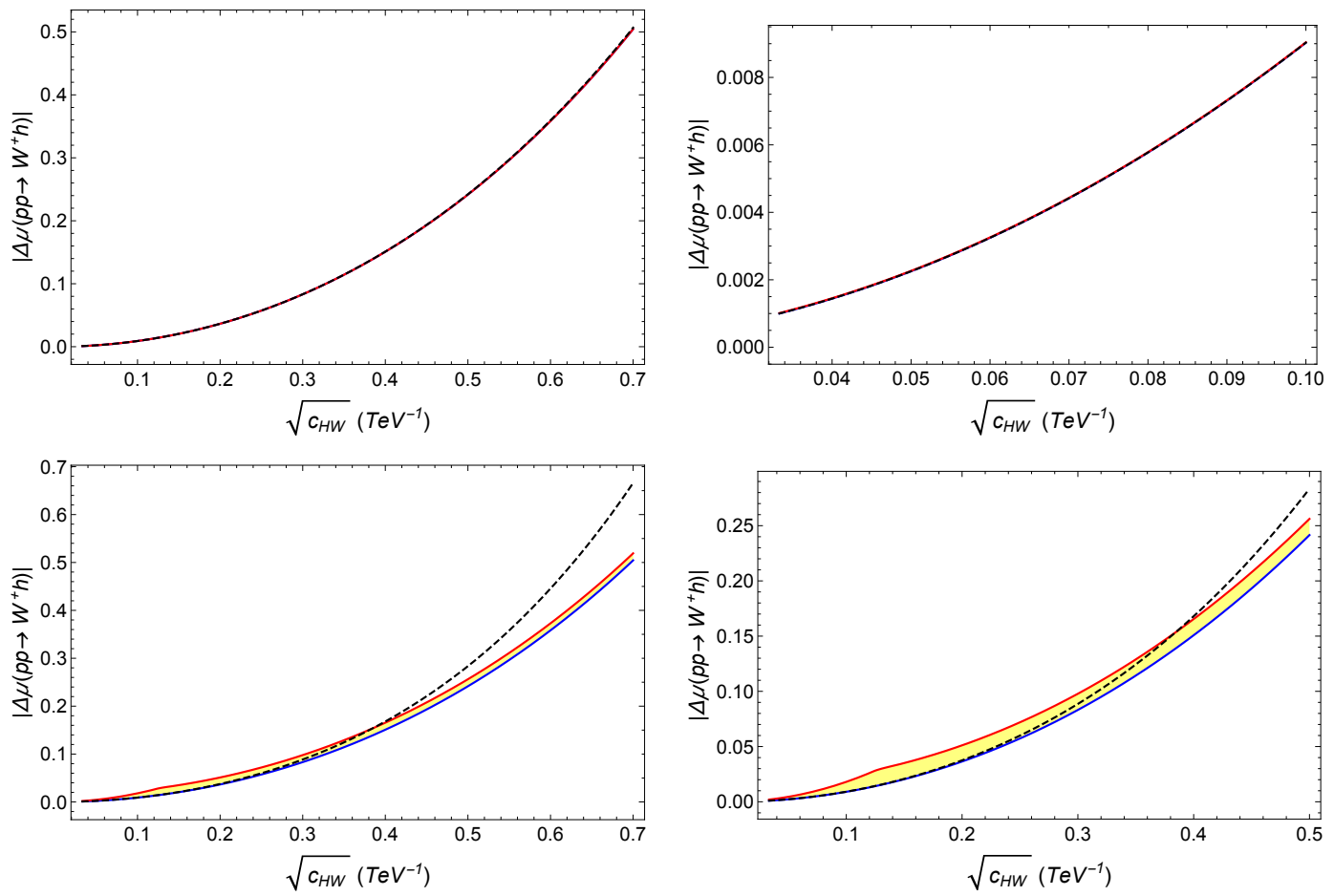

Figure 2. Relative deviation in the inclusive cross section $\sigma\left(p p \rightarrow h W^{+}\right)$at the $\operatorname{LHC}(\sqrt{s}=$ $13 \mathrm{TeV}$ ) from its SM value including dimension- 6 and dimension- 8 effects. The blue line shows the result of including $\mathcal{O}_{H W}$ as the only dimension-6 operator and without considering dimension8 operators. The red line indicates the deviation as a function of $c_{H W}$ including the maximum possible dimension-8 effect consistent with the EFT expansion. The black dashed line shows the result if dimension- 6 and dimension- 8 operator coefficients are equal, $c_{H W}=c_{8, i}$ (i.e. $\Lambda_{8}=\Lambda_{6}$ ). In the top two panels the dimension- 8 coefficients are all equal, while in the bottom two panels we take their magnitudes to be equal but assign their signs to maximize their effects at high $\sqrt{s}$. The left panels shows values of $\sqrt{c_{H W}}$ out to the current 95\% CL limit, which following the global analysis in ref. [90] is $0.631 \mathrm{TeV}^{-1}$. In the right panels we have zoomed in to smaller values of $c_{H W}$ to make the dimension- 8 effects more visible.

The blue line in figure 2 shows the relative deviation in $\sigma\left(p p \rightarrow h W^{+}\right)$from the SM value as a function of $c_{H W}$, neglecting dimension- 8 effects $\left(\Lambda_{8} \rightarrow \infty\right)$. The impact of the dimension- 8 operators can be seen by tracing either vertical or horizontal lines through figure 2. Picking a value of $c_{H W}$ and tracing vertically upwards from the blue line intercepts two lines with different dimension- 8 scenarios. The black dashed line corresponds to the case where dimension- 6 and dimension- 8 operators have the same coefficient, $c_{H W}=c_{8, i}$ or $\Lambda_{6}=\Lambda_{8}$. The red line denotes where $\Lambda_{8}$ has been reduced such that two stringent EFT validity conditions are met: i) the $A_{\mathrm{SM}} \times A_{\text {dim- } 8}$ contribution to the cross section of $\mathcal{O}\left(1 / \Lambda_{8}^{4}\right)$ is greater than the quadratic dimension-8 contribution, $\left|A_{\text {dim- } 8}\right|^{2} \sim \mathcal{O}\left(1 / \Lambda_{8}^{8}\right)$; and ii.) the SM interference with dimension- 6 at $\mathcal{O}\left(1 / \Lambda_{6}^{2}\right)$ is larger than $\mathrm{SM}$ interference with dimension- 8 at $\mathcal{O}\left(1 / \Lambda_{8}^{4}\right)$. The first condition is independent of the dimension- 6 effect, while the latter ties the two terms together and introduces model dependence since in some cases the interference between SM and dimension-6 operators is naturally suppressed. As 
$c_{H W}$ decreases ( $\Lambda_{6}$ increases), this second condition becomes the stronger of the two, causing the tapering in the band of dimension- 8 effects. We also require the condition $A_{\mathrm{SM}} \times A_{\text {dim-6 }}>\left|A_{\text {dim- } 6}\right|^{2}$ in all calculations, which sets the maximum $c_{H W}$ value (the right-hand edge of the plot). The results with and without the validity conditions are shown to demonstrate the range of possible dimension- 8 effects.

Comparing the top and bottom panels of figure 2, the band of dimension- 8 effects is significantly smaller when we choose all dimension- 8 coefficients to have the same sign. The origin of this difference is an accidental cancellation among the dimension- 8 terms when they have the same sign and magnitude. Specifically, the $c_{8,3 Q 1}, c_{8,3 Q 3}$, and $c_{8,3 Q 4}$ terms positively interfere with the SM amplitude, while the $c_{8,3 Q 2}, c_{8, Q W 3}$, and $c_{8, Q W 5}$ terms interfere negatively. The coefficients $c_{8, Q W 3}$ and $c_{8, Q W 5}$ do not appear in eq. (4.4) as they enter the cross section at $O\left(\hat{s}^{0}\right)$, proportional to $\hat{v}^{4} /\left(m_{W}^{2} \Lambda^{4}\right)$. Cancellations between the $\mathcal{O}(\hat{s})$ and $\mathcal{O}\left(\hat{s}^{0}\right)$ pieces are possible since the inclusive production of $W h$ is dominantly near threshold, where $\hat{s} \sim v^{2}$ and thus the two terms are similarly sized. One may think that an accidental cancellation in $A_{\text {dim-8 }}$ can be compensated by lowering $\Lambda_{8}$. However, the $\left|A_{\text {dim- } 8}\right|^{2}$ contribution has no such cancellation, so it overwhelms the $A_{S M} \times A_{\text {dim- } 8}$ piece even at relatively high $\Lambda_{8}$. Thus, the net result of the cancellation in $A_{\text {dim-8 }}$ and our EFT consistency conditions is that the dimension- 8 effects for the positive sign choice are reduced to a sliver. The cancellation in $A_{\text {dim-8 }}$ is broken if we relax the assumption of equal size coefficients or fixed signs. Turning on various combinations of dimension- 8 couplings and adjusting their signs, we find that results similar in size and shape to the bottom panels in figure 2 are far more common; therefore we will use these signs when quantifying the dimension- 8 effects. The net effect of the interference is positive for this choice but negative interference is also possible, in which case the yellow band would lie beneath the blue line.

To get an estimate of how much dimension- 8 operators affect the extraction of the dimension- 6 coefficients, we return to figure 2 and trace horizontally through a fixed value of $\left|\Delta \mu\left(p p \rightarrow h W^{+}\right)\right|$. Let us take $\left|\Delta \mu\left(p p \rightarrow h W^{+}\right)\right|=0.2$ as an example. Extending a horizontal line through that point in the bottom panels, we intersect the red line corresponding to the maximum considered dimension-8 effects at $\sqrt{c_{H W}}=1 /(2.19 \mathrm{TeV})$ and the blue line corresponding to no considered dimension- 8 effects at $\sqrt{c_{H W}}=1 /(2.27 \mathrm{TeV})$. At this level of $\left|\Delta \mu\left(p p \rightarrow h W^{+}\right)\right|$, the relative difference is quite small, $\leq 4 \%$. However, for $\Delta \mu\left(p p \rightarrow h W^{+}\right)=0.05$, the relative size of the dimension- 8 effects in our treatment is $\approx 18 \%$, roughly spanning $\sqrt{c_{H W}}=1 /(4.28 \mathrm{TeV})$ for no dimension- 8 effects to $\sqrt{c_{H W}}=1 /(5.08 \mathrm{TeV})$ including maximal dimension- 8 effects. To see the impact of $\Lambda_{8}=\Lambda_{6}$, rather than the maximal allowed dimension- 8 effect, we repeat the above procedure but look for where a line of constant $\left|\Delta \mu\left(p p \rightarrow h W^{+}\right)\right|$intersects the dashed black line. For $|\Delta \mu(p p \rightarrow W h)|=0.2$, the intersection lies outside of the yellow band, meaning that the $\Lambda_{8}=\Lambda_{6}$ point lies outside our definition of EFT validity. For $\left|\Delta \mu\left(p p \rightarrow h W^{+}\right)\right|=0.05$, the intersection is at $\sqrt{c_{H W}}=1 /(4.36 \mathrm{TeV})$, a $2 \%$ shift from the dimension- 6 value. For reference, ATLAS projects a $W h(\rightarrow \bar{b} b)$ precision at the HL-LHC of $|\Delta \mu| \sim 0.14$ [91], and a global precision including all channels of $<10 \%$ [92]. We emphasize that our results use a single (but representative) sign assignment and equal-magnitude dimension- 8 coefficients, and are therefore only indicative. 

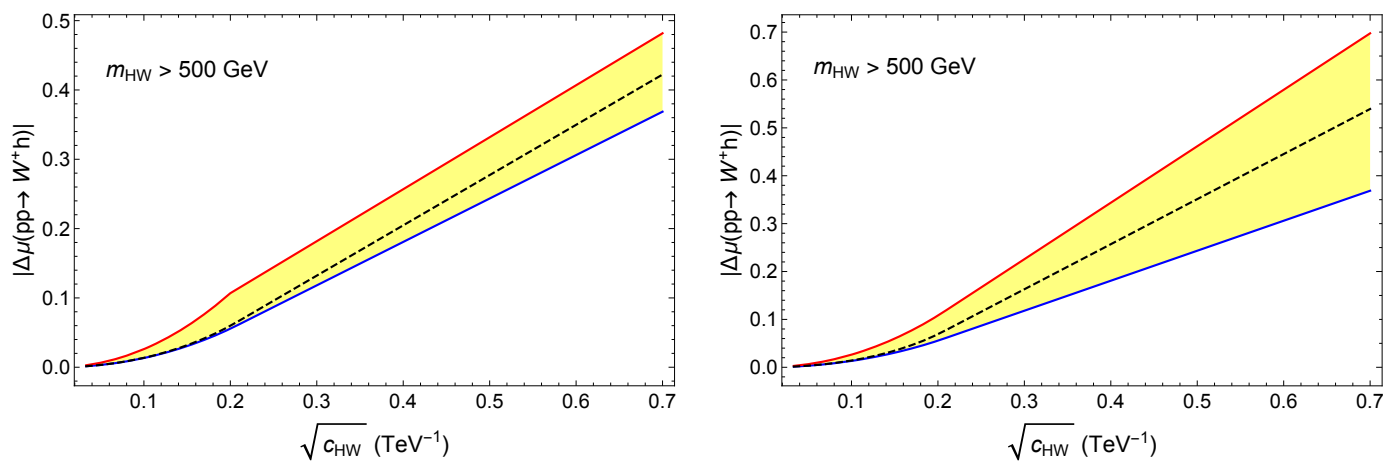

Figure 3. Relative deviation in the high-mass cross section $\sigma\left(p p \rightarrow h W^{+}, m_{H W}>500 \mathrm{GeV}\right)$ at the LHC $(\sqrt{s}=13 \mathrm{TeV})$ from its SM value including dimension- 6 and dimension- 8 effects. In the left panel, all dimension- 8 coefficients are taken to be positive, while in the right panel the signs of the coefficients enhance the impact of dimension- 8 operators on this cross section. The blue, red, and dashed black lines correspond to the same scenarios as in figure 2.

To see how the dimension- 8 operators affect high-scale kinematic regions, we repeat the $\sigma\left(p p \rightarrow h W^{+}\right)$calculation focussing on a region of high invariant mass, $m_{H W} \equiv \sqrt{\hat{s}}>$ $500 \mathrm{GeV}$. The results are shown in figure 3 , both for the case where all dimension- 8 coefficients are positive and for the sign assignment in figure 2. Compared to the inclusive case, the effects of adding dimension- 8 operators are significantly larger and the EFT validity conditions, which must be recalculated for this kinematic region, carve out a different shape. The increased $\hat{s}$ also somewhat disrupts the cancellation in $A_{\text {dim-8 }}$ for the inclusive cross section when all dimension- 8 coefficients are taken to have the same sign. Quantifying the effect, in the mixed-sign case a measurement of $\left|\Delta \mu\left(p p \rightarrow h W^{+}\right)\right|_{m_{H W}>500 \mathrm{GeV}}=0.2$ can be interpreted as $\sqrt{c_{H W}}=1 /(2.32 \mathrm{TeV})$ neglecting dimension- 8 operators and $\sqrt{c_{H W}}=$ $1 /(3.59 \mathrm{TeV})$ including maximal dimension- 8 effects $(\mathrm{a} \sim 55 \%$ difference $)$. For $\Lambda_{8}=\Lambda_{6}$, the effect shrinks to $27 \%\left(\sqrt{c_{H W}}=1 /(2.95 \mathrm{TeV})\right) .{ }^{20}$

The impact of dimension- 8 would be significantly smaller had we neglected the contact terms, illustrating the importance of including all operators that can contribute to a process. This statement is not intended to give the impression that contact terms are special, as the fact that they are the operators with contributions that grow with $\hat{s}$ is an artifact of our use of the Warsaw basis. In other bases, such as the SILH [93] basis, contributions growing with $\hat{s}$ would still be present though not necessarily originating from contact terms. The relative importance of the different operators would also be different in a scenario with unequal coefficients.

The trends exhibited in figures 2 and 3 are not surprising: the more precisely a quantity is measured, the more sensitive it is to higher order corrections; and direct probes of high scales are more sensitive to higher-dimension operators. However, this is the first time dimension-8 effects have been quantified in an LHC process using the complete set of $\left(N_{f}=1\right)$ dimension- 8 operators.

Our analysis has assumed that only $c_{H W}$ is non-zero. This operator enters $\sigma(p p \rightarrow$ $\left.h W^{+}\right)$at $\mathcal{O}\left(\hat{s}^{0}\right)$, whereas other dimension-6 contributions carry different energy depen-

\footnotetext{
${ }^{20}$ For the common-sign case, $\sqrt{c_{H W}}=1 /(3.08 \mathrm{TeV})$ including maximum dimension- 8 effects, and $\sqrt{c_{H W}}=1 /(2.54 \mathrm{TeV})$ for $\Lambda_{8}=\Lambda_{6}$.
} 
dence. We have seen that $c_{H Q}^{(3)}$ enters at $\mathcal{O}(\hat{s})$, while other operators - in particular, operators that only contribute to $p p \rightarrow h W^{+}$via normalization or electroweak inputs enter at $\mathcal{O}\left(\hat{s}^{-1}\right)$. As the current bounds on $c_{H W}, c_{H Q}^{(3)}$, etc. are not radically disparate, it is interesting to investigate the dimension- 8 effects in scenarios with different dimension- 6 energy dependence. The results of repeating the analysis in this section for $c_{H Q}^{(3)}$ or $c_{H \square}$ variations can be found in appendix $\mathrm{F}$.

\section{Conclusions}

In this paper we have evaluated the effect of dimension- 8 operators on two Higgs-boson production cross sections, one inclusive and one in a high- $Q^{2}$ region. For this purpose we have compiled a complete and non-redundant set of dimension- 8 operators involving gauge bosons and at least one Higgs boson, along with the fermionic contact operators necessary to study $h W$ production at the LHC. A vital tool aiding in this construction was the Hilbert series, which tells us how many independent operators exist for each combination of fields, accounting for possible redundancies due to the equations of motion and integration by parts. Applied to the SMEFT, we find 17 independent operators with zero derivatives, 26 with two derivatives, 3 with four derivatives, and 20 operators involving $h, W$ and lefthanded quarks. We have worked with the simplification $N_{f}=1$ for the fermionic operators, which eliminates contributions from four-fermion operators to shifts in the electroweak input parameters.

Through a series of examples, we outlined the steps required to convert between Hilbert series output - the number of invariants and their field and derivative content - to a canonical, phenomenology-ready form including Lorentz and gauge indices. This set of steps is completely general to relativistic EFTs with fields in linear representations of the defining symmetry groups, and is based on a method first proposed in ref. [45]. The most involved step in the translation is the imposition of IBP redundancies, which is performed by constructing a matrix of IBP relations between the operators with all possible derivative partitions and the operators with the same field content but fewer derivatives, and then taking the matrix to row-reduced form. The translation procedure would clearly benefit from automation, especially if one would like to extend it to other sets of observables (see ref. [78] for recent progress in that direction). We have made available the complete set of operators affecting $p p \rightarrow h W$ in FeynRules format in the hope that it will prove useful for future analyses.

We used this framework to study the impact of dimension- 8 operators on the production of a Higgs boson in association with a $W^{+}$boson. This channel provides a good handle on higher dimensional operators due to the kinematic reach of this topology. Higher dimensional operators contribute to the $\bar{q} q W$ vertex, $h W W$ vertex, and $\bar{q} q W h$ contact terms. These contributions accompany different energy dependencies in the cross section. In particular, several contact operators lead to $\sigma\left(p p \rightarrow h W^{+}\right)$contributions that grow with $\hat{s}$. Unlike their counterparts at dimension-6, the dimension- 8 operators that impact $\bar{q} q W h$ do not modify the trilinear $\bar{q} q V$ coupling.

To estimate of the effects of dimension- 8 operators, we studied scenarios where only one dimension- 6 operator coefficient is nonzero and all dimension- 8 operator coefficients 
have equal magnitude but different sign. We quantified the uncertainty on the extracted dimension- 6 coefficient value by drawing contours of constant deviation in $\sigma\left(p p \rightarrow h W^{+}\right)$ and seeing where they intersected the predictions for additional dimension- 6 terms and additional dimensions 6 and 8 terms. Taking $c_{H W} \neq 0$ as the representative dimension- 6 operator and varying the signs of the dimension- 8 operator coefficients, we find the effects of dimension- 8 are typically at the percent level for the inclusive cross section at its currently measured accuracy, growing to $\mathcal{O}(10 \%)$ once we reach $\left|\Delta \mu\left(p p \rightarrow h W^{+}\right)\right|=0.05$. The exact percentage varies depending on whether the dimension- 8 effects act coherently or if there is some cancellation among different operators. We define the maximum size of the dimension- 8 contribution by demanding EFT consistency, including the requirement that the contribution to the cross section linear in the dimension- 8 coefficients makes a larger contribution to the cross section than the quadratic dimension- 8 terms. If we focus on high-mass kinematic regions, the effects of dimension- 8 operators become much larger. For example, for the coefficient set we use, dimension- 8 operators shift the dimension- 6 coefficient implied by $\left|\Delta \mu\left(p p \rightarrow h W^{+}\right)_{m_{H W}>500 \mathrm{GeV}}\right|=0.2$ by $55 \%$. In order to carry out these phenomenological studies, we performed canonical normalization and electroweak input procedures including dimension-8 effects (see appendix D).

This study and its companion implementation in FeynRules (see appendix A) open the possibility for experimental collaborations and theoretical analyses to assign a systematic uncertainty to the effect of dimension- 8 operators. Within the context of $p p \rightarrow h W^{+}$, it would be interesting to carry out uncertainty estimates for dimension- 6 operators other than $c_{H W}, c_{H Q}^{(3)}$, or $c_{H \square}$, or to more thoroughly explore the effects of correlations and cancellations among different operators. This work also allows theoretical studies of the interplay of dimension- 8 operators with specific types of new physics, for example CP-odd Higgs couplings at dimension- 6 .

Moving beyond $p p \rightarrow h W^{+}$, the logical next step is to extend the current FeynRules implementation to more operators and study the impact of dimension- 8 effects on Higgs production in association with a $Z$ boson or with top quarks. It would also be interesting to add pure gauge dimension- 8 operators (such as those in [40]) which would affect di-boson and tri-boson production, and to relate these to the anomalous trilinear and quadrilinear gauge couplings (aTGC and aQGC), see for example references [94, 95].

Note added. As this paper was being completed, ref. [78] appeared demonstrating a similar method for explicitly constructing non-redundant sets of higher dimensional operators in the SMEFT and beyond. That reference includes a software package automatizing the required steps.

\section{Acknowledgments}

The work of VS is supported by the Science Technology and Facilities Council (STFC) under grant number ST/P000819/1. The work of AM was partially supported by the National Science Foundation under Grant No. PHY-1520966. We would like to thank Andrea Banfi for discussions on the jet-veto effects in $W h$ production. AM would like to thank Landon Lehman for his input during the initial stages of the project. 


\section{A Implementation in FeynRules}

In this section we briefly discuss the implementation in FeynRules. Two .fr files are attached to this paper as supplementary material, and will be available shortly in the model database in their webpage (http://feynrules.irmp.ucl.ac.be). Both files contain the operators in appendix B for dimension 6 and tables $4-7$ for dimension 8 . The operator coefficients in the .fr files have the same name convention we have used in the text, e.g. c8HD as the coefficient of $\mathcal{O}_{8, H D}$. Within the files, the operators are grouped according to whether they influence $p p \rightarrow h W^{+}$, and the contact operators have been split into individual modules to speed up the code. The difference between the two files is whether or not the canonical normalization and electroweak input procedures described in appendix D have been carried out. The procedures are needed to consistently include dimension8 effects but they significantly slow down the running of the current incarnation of the .fr files, so we have provided a version omitting that step. We have made three other simplifications: 1.) as we are working with the $N_{f}=1$ SMEFT, there are no four-fermion contributions to $G_{F}, 2$.) as our focus here is on $h W$ production we have neglected the $\mathcal{O}\left(\Lambda^{-4}\right)$ difference between $\sin ^{2} \bar{\theta}$ and $\sin ^{2} \theta_{Z}$ in the coupling of $Z$ bosons to fermions, and 3.) we have omitted all CP-violating dimension- 8 fermionic operators. We will address these shortcuts in future versions of the .fr and plan to extend the set of translated operators to explore $p p \rightarrow Z h$ and $p p \rightarrow V V$. To ensure that events with these .fr include dimension-8 interference effects but not $\left|A_{\text {dim-8 } 8}\right|^{2}$, one should generate events with the suffix NP^2<=2.

\section{B Dimension-6 operators}

\begin{tabular}{|c|c|c|c|}
\hline $\mathcal{O}_{H}$ & $\left(H^{\dagger} H\right)^{3}$ & $\mathcal{O}_{H \tilde{W}}$ & $\delta_{I J}\left(H^{\dagger} H\right) W_{\mu \nu}^{I} \widetilde{W}_{\mu \nu}^{J}$ \\
$\mathcal{O}_{H \square}$ & $\left(H^{\dagger} H\right) D^{2}\left(H^{\dagger} H\right)$ & $\mathcal{O}_{H B}$ & $\left(H^{\dagger} H\right) B_{\mu \nu} B_{\mu \nu}$ \\
$\mathcal{O}_{H D}$ & $\left(D_{\mu} H^{\dagger} H\right)\left(H^{\dagger} D_{\mu} H\right)$ & $\mathcal{O}_{H \tilde{B}}$ & $\left(H^{\dagger} H\right) B_{\mu \nu} \widetilde{B}_{\mu \nu}$ \\
$\mathcal{O}_{H G}$ & $\delta_{A B}\left(H^{\dagger} H\right) G_{\mu \nu}^{A} G_{\mu \nu}^{B}$ & $\mathcal{O}_{H W B}$ & $\delta_{I J}\left(H^{\dagger} \tau^{I} H\right) W_{\mu \nu}^{J} B_{\mu \nu}$ \\
$\mathcal{O}_{H \tilde{G}}$ & $\delta_{A B}\left(H^{\dagger} H\right) G_{\mu \nu}^{A} \widetilde{G}_{\mu \nu}^{B}$ & $\mathcal{O}_{H \tilde{W} B}$ & $\delta_{I J}\left(H^{\dagger} \tau^{I} H\right) \widetilde{W}_{\mu \nu}^{J} B_{\mu \nu}$ \\
$\mathcal{O}_{H W}$ & $\delta_{I J}\left(H^{\dagger} H\right) W_{\mu \nu}^{I} W_{\mu \nu}^{J}$ & $\mathcal{O}_{H Q}^{(1)}$ & $i\left(Q^{\dagger} \bar{\sigma}^{\mu} Q\right)\left(H^{\dagger} \overleftrightarrow{D}^{\mu} H\right)$ \\
& & $\mathcal{O}_{H Q}^{(3)}$ & $i\left(Q^{\dagger} \bar{\sigma}^{\mu} \tau^{I} Q\right)\left(H^{\dagger} \overleftrightarrow{D}^{\mu} \tau^{I} H\right)$ \\
\hline
\end{tabular}

Table 8. The thirteen dimension- 6 operators included for comparison with dimension- 8 effects. As described in the text, we work with the Warsaw basis.

\section{Other examples}

In this appendix we give two further examples to illustrate the conversion from the Hilbert series output to EFT operators in their canonical form. 


\section{C.1 Example: $2\left(H^{\dagger} H\right)^{2} W_{L}^{2}$}

In this example we will demonstrate the procedures for handling multiple operators and non-trivial $\mathrm{SU}(2)_{w}$ contractions. The coefficient of the Hilbert series output for the operator $2\left(H^{\dagger} H\right)^{2} W_{L}^{2}$ indicates that we need to find two invariants. The Higgs field portion of $\left(H^{\dagger} H\right)^{2} W_{L}^{2}$ is identical to the example in section 2.1.1, so the Higgs group-theory decomposition is identical to eq. (2.5). The remaining object, $W_{L}$, is bosonic, so $\left(W_{L}\right)^{2}$ must be symmetric. However, $W_{L}$ transforms under both $\mathrm{SU}(2)_{L}$ and $\mathrm{SU}(2)_{w}$ so there are more ways to form a symmetrized product. Specifically, $W_{L}^{2}$ can be overall symmetric if it is either symmetric in both $\left(\mathrm{SU}(2)_{L}, \mathrm{SU}(2)_{w}\right)$ indices or antisymmetric in both: ${ }^{21}$

$$
\begin{aligned}
W_{L}^{2} & =(1,0 ; 1)_{\mathrm{symm}}^{2}=(0 \oplus 1 \oplus 2,0 ; 0 \oplus 1 \oplus 2)_{\mathrm{symm}}=(0 \oplus 2,0 ; 0 \oplus 2)+(1,0 ; 1) \\
& =((0,0 ; 0)+(2,0 ; 0)+(0,0 ; 2)+(2,0 ; 2))+(1,0 ; 1) .
\end{aligned}
$$

We know the Higgs part of the operators is a Lorentz singlet, so only Lorentz-singlet $W_{L}^{2}$ options can make potential invariants: $(0,0 ; 0)$ and $(0,0 ; 2)$. Adding in the Higgs fields,

$$
\begin{aligned}
H^{2}\left(H^{\dagger}\right)^{2}\left(W_{L}^{2}\right) & =((0,0 ; 1) \otimes(0,0 ; 1)) \otimes(0,0 ; 0 \oplus 2) \\
& =(0,0 ; 0 \oplus 2) \otimes(0,0 ; 0 \oplus 2),
\end{aligned}
$$

we can pick out the two invariants. One invariant comes from the product of the $\mathrm{SU}(2)_{w}$ singlet element of $\left(H^{\dagger} H\right)^{2}$ with the $\mathrm{SU}(2)_{w}$ singlet piece of $W_{L}^{2}$, while the other comes from the $\mathrm{SU}(2)_{w}$ spin-2 (symmetric tensor) piece of $\left(H^{\dagger} H\right)^{2}$ with the corresponding piece of $W_{L}^{2}$.

For the product of $\mathrm{SU}(2)_{w}$ singlets, the $H^{2}$ and $\left(H^{\dagger}\right)^{2}$ terms are contracted together, ${ }^{22}$ as are the two $W_{L}$ fields,

$$
\left(\epsilon H^{\dagger} H\right)^{2} W_{L, \mu \nu}^{I} W_{L}^{I, \mu \nu}
$$

Similarly, to form the invariant from $\mathrm{SU}(2)_{w} 2 \otimes 2$, we want to contract the symmetric product of the $H^{2},\left(H^{\dagger}\right)^{2}$ triplets with the symmetric product of $W_{L}$ triplets

$$
\left(\operatorname{Tr}\left(H \tau^{A} \epsilon H\right) \operatorname{Tr}\left(H^{\dagger} \tau^{B} \epsilon H^{\dagger}\right)+A \leftrightarrow B\right)\left(W_{L, \mu \nu}^{A} W_{L}^{B, \mu \nu}+A \leftrightarrow B\right),
$$

where the $\epsilon$ in the $H^{2}$ product appears (as in eq. (C.2)) because $H^{\dagger}$ is a 2 of $\mathrm{SU}(2)_{w}$. The two terms are identical, so the operator collapses to

$$
\operatorname{Tr}\left(H \tau^{A} \epsilon H\right) \operatorname{Tr}\left(H^{\dagger} \tau^{B} \epsilon H^{\dagger}\right) W_{L, \mu \nu}^{A} W_{L}^{B, \mu \nu} .
$$

\footnotetext{
${ }^{21}$ Symmetrizing will depend on the representation we are working with; for $\mathrm{SU}(2)$ triplets the symmetric combinations of $X_{A} Y_{B}$ (spin-0) are with $\delta^{A B}$ or the two-index symmetric tensor $X_{\{A} Y_{B\}}$ (spin-2), while the antisymmetric case is the antisymmetric tensor $X_{(A} Y_{B)}=$ vector, $\epsilon^{A B C} X_{A} Y_{B}$ (spin-1). However if we deal with $\mathrm{SU}(2)$ doublets, spin- 0 is the antisymmetric combination $\epsilon^{i j} x_{i} y_{j}$ while spin-1 (vector) is the symmetric case $x_{\{i} y_{j\}}$.

${ }^{22}$ Which we can form either by inspection, or by taking the singlet product of the $H^{2}$ triplet and $\left(H^{\dagger}\right)^{2}$ triplet, then simplifying via the identity $\tau_{i j}^{A} \tau_{l m}^{A}=2 \delta_{i m} \delta_{j l}-\delta_{i j} \delta_{l m}$.
} 
Technically, to form a true spin-2 representation from the product of spin-1 (triplet) representations, we should have subtracted a piece proportional to $\delta_{A B}$. However, as a $\delta_{A B}$ would reduce the operator in eq. (C.3) to the form eq. (C.2), we can just absorb that contribution into the coefficient of eq. (C.2). Repeating these steps for the hermitian conjugate term $\left(H^{\dagger} H\right)^{2} W_{R}^{2}$ gets us terms analogous to eqs. (C.2) and (C.3) but with $W_{L} \rightarrow W_{R}$. Finally, as with the example in section 2.1.1, we can separate the real and imaginary pieces into independent operators by considering linear combinations of the $W_{L}$ and $W_{R}$ forms. Written in terms of $W, \tilde{W}$ and with $H^{\dagger}$ as a $\overline{2}$, the four operators are:

$$
\begin{aligned}
\mathcal{O}_{8, H W} & =\left(H^{\dagger} H\right)^{2} W_{\mu \nu}^{I} W^{I, \mu \nu}, & \mathcal{O}_{8, H \tilde{W}} & =\left(H^{\dagger} H\right)^{2} W_{\mu \nu}^{I} \tilde{W}^{I, \mu \nu} \\
\mathcal{O}_{8, H W 2} & =\left(H^{\dagger} \tau^{I} H\right)\left(H^{\dagger} \tau^{J} H\right) W_{\mu \nu}^{I} W^{J, \mu \nu}, & \mathcal{O}_{8, H \tilde{W} 2} & =\left(H^{\dagger} \tau^{I} H\right)\left(H^{\dagger} \tau^{J} H\right) W_{\mu \nu}^{I} \tilde{W}^{J, \mu \nu} .
\end{aligned}
$$

\section{C.2 Example: $4 D\left(Q^{\dagger} Q\left(H^{\dagger} H\right)^{2}\right)$}

The goal of this example is to show how to manipulate operators with fermions and operators with a single derivative. As discussed in section 2.1, the first step is to enumerate the ways we can partition the derivative. The derivative cannot act on $Q$ or $Q^{\dagger}$ since $D Q, D Q^{\dagger}$ will transform under both Lorentz $\mathrm{SU}(2)_{L}$ and $\mathrm{SU}(2)_{R}$, while the other object in a non-trivial Lorentz representation only transforms under one of the two. The derivative can therefore either act on $H$ or $H^{\dagger}$, so let us begin with $D H$. There are no repeated fermion fields so we do not need to worry about anytisymmetrization, and we can ignore $\mathrm{SU}(3)$ since it is clear that we only want the color-singlet portion of $Q Q^{\dagger}$. Sticking with just Lorentz and $\mathrm{SU}(2)_{w}$ indices and grouping terms conveniently:

$$
\begin{aligned}
\left(Q^{\dagger} Q\right)(H D H)\left(H^{\dagger}\right)^{2} & =\left(\frac{1}{2}, \frac{1}{2} ; 0 \oplus 1\right) \times\left(\frac{1}{2}, \frac{1}{2} ; 0 \oplus 1\right) \times(0,0 ; 1) \\
& =(0,0 ; 0 \oplus 0 \oplus 1 \oplus 1 \oplus 1 \oplus 2) \times(0,0 ; 1) .
\end{aligned}
$$

This gives three terms, roughly: i.) the triplet of $Q^{\dagger} Q$ contracted with the $H^{\dagger}$ triplet, ii.) the triplet of $H D H$ contracted with the $H^{\dagger}$ triplet, or iii.) contracting all three triplets with an $\epsilon^{I J K}$. Using $\mathrm{SU}(2)_{w}$ indices the terms are:

$$
\left(Q^{\dagger} Q\right)_{\{a b\}}(H D H)\left(H^{\dagger}\right)_{\{c d\}}^{2} \epsilon^{a c} \epsilon^{b d}=\operatorname{Tr}\left(Q^{\dagger} \tau^{I} \epsilon Q\right)(H D H) \operatorname{Tr}\left(H^{\dagger} \tau^{J} \epsilon H^{\dagger}\right) \delta_{I J}
$$

iii.) $\left(Q^{\dagger} Q\right)_{\{a b\}}(H D H)_{\{i j\}}\left(H^{\dagger}\right)_{\{c d\}}^{2} \epsilon^{a i} \epsilon^{b c} \epsilon^{j d}=\operatorname{Tr}\left(Q^{\dagger} \tau^{I} \epsilon Q\right) \operatorname{Tr}\left(H \tau^{J} \epsilon D H\right) \operatorname{Tr}\left(H^{\dagger} \tau^{K} \epsilon H^{\dagger}\right) \epsilon_{I J K}$.

Replacing $D H \rightarrow D H^{\dagger}$ we have three more terms (iv., v., iv.), so a total of six. However, if we IBP on term ii.) above, we get

$$
\begin{aligned}
& \left(Q^{\dagger} Q\right)(H D H)_{\{a b\}}\left(H^{\dagger}\right)_{\{c d\}}^{2} \epsilon^{a c} \epsilon^{b d} \rightarrow \\
& \text { (total deriv.) }-\left(Q^{\dagger} Q\right)(D H H)_{\{a b\}}\left(H^{\dagger}\right)_{\{c d\}}^{2} \epsilon^{a c} \epsilon^{b d}-2\left(Q^{\dagger} Q\right)\left(H^{2}\right)_{\{a b\}}\left(D H^{\dagger} H^{\dagger}\right)_{\{c d\}} \epsilon^{a c} \epsilon^{b d},
\end{aligned}
$$


where we have ignored any terms with the derivative on the $Q, Q^{\dagger}$ since we know they cannot yield invariants. Since the $\mathrm{SU}(2)_{w}$ indices are all symmetric, the second term is the same as the original operator and the factor of 2 arises since differentiating either of the $H^{\dagger}$ gives the same result. Ignoring the total derivative and rearranging, we find IBP gives:

$$
\left(Q^{\dagger} Q\right)(H D H)_{\{a b\}}\left(H^{\dagger}\right)_{\{c d\}}^{2} \epsilon^{a c} \epsilon^{b d} \rightarrow\left(Q^{\dagger} Q\right)\left(H^{2}\right)_{\{a b\}}\left(H^{\dagger} D H^{\dagger}\right)_{\{c d\}} \epsilon^{a c} \epsilon^{b d} .
$$

This tells us that operators ii.) and v.) are not independent since we can always IBP on one to generate the other. The same manipulations work for operators iii.) and vi.), reducing the number of independent invariants to 4 . The same trick cannot be applied to operators i.) and iv.), since if we remove the derivative from $(H D H)$ Bose symmetrization eliminates the operator. ${ }^{23}$ The only surviving term in the IBP is when we shift the derivative from one $H$ to the other, getting us back operator i.). The 4 independent invariants are then

$$
\begin{array}{lll}
\text { i. }) & \operatorname{Tr}\left(Q^{\dagger} \bar{\sigma}^{\mu} \tau^{I} \epsilon Q\right)\left(H D_{\mu} H\right) \operatorname{Tr}\left(H^{\dagger} \tau^{J} \epsilon H^{\dagger}\right) \delta_{I J} & \\
\text { ii. }) & \operatorname{Tr}\left(Q^{\dagger} \bar{\sigma}^{\mu} \tau^{I} \epsilon Q\right)\left(H^{\dagger} D_{\mu} H^{\dagger}\right) \operatorname{Tr}\left(H \tau^{J} \epsilon H\right) \delta_{I J} & \\
\text { iii. }) & \left(Q^{\dagger} \bar{\sigma}^{\mu} Q\right) \operatorname{Tr}\left(H \tau^{I} \epsilon D_{\mu} H\right) \operatorname{Tr}\left(H^{\dagger} \tau^{J} \epsilon H^{\dagger}\right) \delta_{I J} & \text { or } \quad D H \rightarrow D H^{\dagger} \\
\text { iv. }) & \operatorname{Tr}\left(Q^{\dagger} \bar{\sigma}^{\mu} \tau^{I} \epsilon Q\right) \operatorname{Tr}\left(H \tau^{J} \epsilon D_{\mu} H\right) \operatorname{Tr}\left(H^{\dagger} \tau^{K} \epsilon H^{\dagger}\right) \epsilon_{I J K} & \text { or } \quad D H \rightarrow D H^{\dagger}
\end{array}
$$

We can utilize Fierz rearrangement identities [96] to convert these into the forms shown in table 7 .

\section{Electroweak inputs and field redefinitions}

Expanded out to dimension-8, the electroweak sector of the SMEFT is a function of the gauge couplings, the Higgs quartic and vev, and the coefficients of the dimension- 6 and -8 operators. In this appendix, we relate combinations of these inputs to precisely measured quantities. The relationships are well known in the SM, and have been worked out previously for the dimension-6 SMEFT [34, 73, 74]. The same methods are applied here, extended to include dimension-6-squared terms and linear dimension- 8 terms as they are the same order in $1 / \Lambda$. As explained in the text, we work in the Warsaw basis throughout.

Only a subset of our operators are important for setting the EW inputs. From dimension 6 they are $\mathcal{O}_{H}, \mathcal{O}_{H \square}, \mathcal{O}_{H D}, \mathcal{O}_{H B}, \mathcal{O}_{H W}, \mathcal{O}_{H W B}$, while those from dimension-8 are $\mathcal{O}_{8, H}$, $\mathcal{O}_{8, H B}, \mathcal{O}_{8, H W B}, \mathcal{O}_{8, H W}, \mathcal{O}_{8, H W 2}, \mathcal{O}_{8, H D}, \mathcal{O}_{8, H D 2}$. In total, the EW sector at this order is a function of 17 inputs (13 operator coefficients, 2 gauge couplings, 1 quartic and 1 vev):

$$
\begin{aligned}
\mathcal{L}_{E W, S M}= & -\frac{1}{4} W_{\mu \nu}^{I} W^{I \mu \nu}-\frac{1}{4} B_{\mu \nu} B^{\mu \nu}+\left(D_{\mu} H^{\dagger}\right)\left(D^{\mu} H\right)-\lambda\left(H^{\dagger} H-\frac{v_{0}^{2}}{2}\right)^{2}, \\
\mathcal{L}_{E W, 6}= & \frac{c_{H}}{\Lambda^{2}}\left(H^{\dagger} H\right)^{3}+\frac{c_{H \square}}{\Lambda^{2}}\left(H^{\dagger} H\right) \square\left(H^{\dagger} H\right)+\frac{c_{H D}}{\Lambda^{2}}\left(\left(D_{\mu} H^{\dagger}\right) H\right)\left(H^{\dagger} D^{\mu} H\right) \\
& +\frac{c_{H W}}{\Lambda^{2}}\left(H^{\dagger} H\right) W_{\mu \nu}^{I} W^{I \mu \nu}+\frac{c_{H B}}{\Lambda^{2}}\left(H^{\dagger} H\right) B_{\mu \nu} B^{\mu \nu}+\frac{c_{H W B}}{\Lambda^{2}}\left(H^{\dagger} \tau^{I} H\right) B_{\mu \nu} W^{I \mu \nu}, \\
\mathcal{L}_{E W, 8}= & \frac{c_{8, H}}{\Lambda^{4}}\left(H^{\dagger} H\right)^{4}+\frac{c_{8, H B}}{\Lambda^{4}}\left(H^{\dagger} H\right)^{2} B_{\mu \nu} B^{\mu \nu}+\frac{c_{8, H W B}}{\Lambda^{4}}\left(H^{\dagger} H\right)\left(H^{\dagger} \tau^{I} H\right) B_{\mu \nu} W^{I \mu \nu} \\
& +\frac{c_{8, H W}}{\Lambda^{4}}\left(H^{\dagger} H\right)^{4} W_{\mu \nu}^{I} W^{I \mu \nu}+\frac{c_{8, H W 2}}{\Lambda^{4}}\left(H^{\dagger} \tau^{I} H\right)\left(H^{\dagger} \tau^{J} H\right) W_{\mu \nu}^{I} W^{J \mu \nu} \\
& +\frac{c_{8, H D}}{\Lambda^{4}}\left(H^{\dagger} H\right)^{2}\left(D_{\mu} H^{\dagger} D^{\mu} H\right)+\frac{c_{8, H D 2}}{\Lambda^{4}}\left(H^{\dagger} H\right)\left(H^{\dagger} \tau^{I} H\right)\left(D_{\mu} H^{\dagger} \tau^{I} D^{\mu} H\right) .
\end{aligned}
$$

\footnotetext{
${ }^{23}$ That is, we go from $\epsilon^{i j} H_{i} D H_{j}$ to $\epsilon^{i j} H_{i} H_{j}=0$.
} 
We set the EW inputs using the $\left\{\alpha_{e w}, M_{Z}^{2}, G_{F}\right\}$ scheme, i.e. we solve for the gauge couplings and Higgs vev in terms of these observables and the coefficients of dimensions 6 and 8. To determine the Higgs quartic coupling we supplement our inputs with the measured Higgs mass, $M_{H}^{2}$. However, before we can relate the EW (and Higgs) inputs to observables calculated in the SMEFT theory, we need to bring eq. (D.1) into canonical form.

The first step is to expand the Higgs field about its vacuum expectation value. In the presence of higher dimensional operators, the minimum of the Higgs potential is no longer at $v_{0}$ but instead at

$$
\langle h\rangle=v_{0}\left(1+\frac{3 c_{H} v_{0}^{2}}{8 \lambda \Lambda^{2}}+\frac{v_{0}^{4}\left(63 c_{H}^{2}+32 c_{8, H} \lambda\right)}{128 \lambda^{2} \Lambda^{4}}\right) \equiv v_{T},
$$

where $\lambda$ is the SM quartic. In unitary gauge the Higgs field is expanded as

$$
H=\frac{1}{\sqrt{2}}\left(\begin{array}{c}
0 \\
\left(1+c_{H, \text { kin }}\right) h+v_{T}
\end{array}\right)
$$

where $c_{H \text {,kin }}$ is the correction to canonically normalize the Higgs field, carried out to $\mathcal{O}\left(1 / \Lambda^{4}\right): c_{H, \text { kin }}=\frac{v_{T}^{2}}{4 \Lambda^{2}}\left(4 c_{H \square}-c_{H D}\right)+\frac{v_{T}^{4}}{32 \Lambda^{4}}\left(3\left(c_{H D}-4 c_{H \square}\right)^{2}-4 c_{8, H D}-4 c_{8, H D 2}\right)$. Notice that the $1 / \Lambda^{4}$ pieces of $c_{H, \text { kin }}$ and $v_{T}$ contain dimension- 8 effects and effects from $(\text { dimension } 6)^{2}$.

The next step is to canonically normalize the gauge fields: $B_{\mu} \rightarrow\left(1+c_{B, \mathrm{kin}}\right) \bar{B}_{\mu}, W_{\mu}^{a} \rightarrow$ $\left(1+c_{W, \text { kin }}^{a}\right) \bar{W}_{\mu}^{a}$ (barred fields are canonical). We can simultaneously redefine the gauge couplings to compensate for these changes, $g_{1} \rightarrow \bar{g}_{1} /\left(1+c_{B, \text { kin }}\right), g_{2} \rightarrow \bar{g}_{2} /\left(1+c_{W \text {,kin }}\right)$, which has the effect that $g_{1} B_{\mu}=\bar{g}_{1} \bar{B}_{\mu}$, etc., so that renormalizable gauge interactions in the dimension-8 SMEFT have the same form as the SM, but with barred couplings and fields. There is one subtlety here compared to dimension 6: the factor $c_{W \text {,kin }}^{a}$ is no longer universal for all $W^{a}$ as a consequence of $\mathcal{O}_{8, H W 2}$. We can only rescale $g_{2}$ once, so we must choose whether to absorb $c_{W^{ \pm}, \text {kin }}$ or $c_{W^{3} \text {,kin }}$. Choosing $g_{2} \rightarrow \bar{g}_{2} /\left(1+c_{W^{ \pm}, \text {kin }}\right)$, the neutral current at dimension- 8 will no longer have the same form (in barred couplings and fields) as the SM [97]. Explicitly:

$$
\begin{aligned}
c_{W^{ \pm}, \mathrm{kin}} & =\frac{v_{T}^{2}}{\Lambda^{2}} c_{H W}+\frac{v_{T}^{4}}{2 \Lambda^{4}}\left(3 c_{H W}^{2}+c_{8, H W}\right) \\
c_{W^{3}, \mathrm{kin}} & =\frac{v_{T}^{2}}{\Lambda^{2}} c_{H W}+\frac{v_{T}^{4}}{2 \Lambda^{4}}\left(3 c_{H W}^{2}+c_{8, H W}+c_{8, H W 2}\right) \\
c_{B, \mathrm{kin}} & =\frac{v_{T}^{2}}{\Lambda^{2}} c_{H B}+\frac{v_{T}^{4}}{2 \Lambda^{4}}\left(3 c_{H B}^{2}+c_{8, H B}\right) .
\end{aligned}
$$

Next, we must diagonalize the kinetic and mass terms for the neutral gauge fields. This can be done following refs. [73, 97],

$$
\begin{aligned}
\left(\begin{array}{c}
\bar{W}_{\mu}^{3} \\
\bar{B}_{\mu}
\end{array}\right) & =\left(\begin{array}{ll}
X_{11} & X_{12} \\
X_{12} & X_{11}
\end{array}\right)\left(\begin{array}{cc}
\cos \bar{\theta} & \sin \bar{\theta} \\
-\sin \bar{\theta} & \cos \bar{\theta}
\end{array}\right)\left(\begin{array}{c}
\bar{Z}_{\mu} \\
\bar{A}_{\mu}
\end{array}\right) \\
X_{11} & =1+\frac{v_{T}^{4}}{8 \Lambda^{4}}\left(3 c_{H W B}^{2}\right) \\
X_{12} & =-\frac{v_{T}^{2}}{2 \Lambda^{2}} c_{H W B}-\frac{v_{T}^{4}}{4 \Lambda^{4}}\left(2 c_{H W B}\left(c_{H B}+c_{H W}\right)+c_{8, H W B}\right),
\end{aligned}
$$


where $\sin \bar{\theta}$ and $\cos \bar{\theta}$ are defined in terms of $v_{T}$, the barred couplings $\bar{g}_{1}, \bar{g}_{2}$ and other dimension- 6 and dimension- 8 coefficients:

$$
\begin{aligned}
& \cos \bar{\theta}= \frac{\bar{g}_{2}}{\sqrt{\bar{g}_{1}^{2}+\bar{g}_{2}^{2}}}\left(1+\frac{v_{T}^{2}}{\Lambda^{2}} \frac{c_{H W B}}{2} \frac{\bar{g}_{1}}{\bar{g}_{2}} \frac{\bar{g}_{1}^{2}-\bar{g}_{2}^{2}}{\bar{g}_{1}^{2}+\bar{g}_{2}^{2}}+\frac{v_{T}^{4}}{8 \Lambda^{4}} \frac{\bar{g}_{1}}{\bar{g}_{2}\left(\bar{g}_{2}^{2}+\bar{g}_{1}^{2}\right)^{2}} \times\right. \\
&\left(2 c_{8, H W B}\left(\bar{g}_{1}^{4}-\bar{g}_{2}^{4}\right)+4 c_{8, H W 2} \bar{g}_{2} \bar{g}_{1}\left(\bar{g}_{2}^{2}+\bar{g}_{1}^{2}\right)+4 c_{H W B}\left(c_{H W}+c_{H B}\right)\left(\bar{g}_{1}^{4}-\bar{g}_{2}^{2}\right)-\right. \\
& \sin \bar{\theta}=\left.\frac{\bar{g}_{1}^{2}}{\sqrt{\bar{g}_{1}^{2}+\bar{g}_{2}^{2}}}\left(1-\frac{\bar{g}_{2}}{\bar{g}_{1}}\left(\bar{g}_{2}^{4}-6 \bar{g}_{1}^{2} \bar{g}_{2}^{2}+5 \bar{g}_{1}^{4}\right)\right)\right) \\
&\left(2 c_{8, H W B} \frac{c_{H W B}}{2} \frac{\bar{g}_{2}}{\bar{g}_{1}} \frac{\bar{g}_{1}^{2}-\bar{g}_{2}^{2}}{\bar{g}_{1}^{2}+\bar{g}_{2}^{2}}+\frac{v_{T}^{4}}{8 \Lambda^{4}} \frac{\bar{g}_{2}}{\bar{g}_{1}\left(\bar{g}_{1}^{2}+\bar{g}_{2}^{2}\right)^{2}} \times\right. \\
& c_{H W B}^{2} \bar{g}_{1} c_{8, H} \bar{g}_{2} \\
&\left.\left.\bar{g}_{1}^{4}-6 \bar{g}_{1}^{2} \bar{g}_{2}^{2}\left(\bar{g}_{1}^{2}+5 \bar{g}_{2}^{2}\right)+4 c_{H W B}\left(c_{H W}+c_{H B}\right)\right)\right) .
\end{aligned}
$$

From the diagonal form, we can read off the gauge-boson masses:

$$
\begin{aligned}
m_{W}^{2}= & \frac{\bar{g}_{2}^{2} v_{T}^{2}}{4}+\frac{\bar{g}_{2}^{2} v_{T}^{6}}{16 \Lambda^{4}}\left(c_{8, H D}-c_{8, H D 2}\right) \\
m_{Z}^{2}= & \frac{v_{T}^{2}\left(\bar{g}_{1}^{2}+\bar{g}_{2}^{2}\right)}{4}+\frac{v_{T}^{2}}{8 \Lambda^{2}}\left(c_{H D}\left(\bar{g}_{1}^{2}+\bar{g}_{2}^{2}\right)+4 c_{H W B} \bar{g}_{2} \bar{g}_{1}\right) \\
& +\frac{v_{T}^{6}}{16 \Lambda^{4}}\left(( \overline { g } _ { 1 } ^ { 2 } + \overline { g } _ { 2 } ^ { 2 } ) \left(c_{8, H D}+c_{8, H D 2}\right.\right. \\
& \left.\left.\quad+4 c_{H W B}^{2}\right)+\overline{4} g_{1} \bar{g}_{2}\left(c_{8, H W B}+c_{H W B}\left(2 c_{H B}+2 c_{H W}+c_{H D}\right)\right)+4 \bar{g}_{2}^{2} c_{8, H W 2}\right) .
\end{aligned}
$$

Expanding the covariant derivative and going to diagonal form, we can extract the couplings to the photon, $Z$ and $W$ bosons:

$$
D_{\mu}=\partial_{\mu}+i \frac{\bar{g}_{2}}{\sqrt{2}}\left(\bar{W}_{\mu}^{+} \tau^{+}+\bar{W}_{\mu}^{-} \tau^{-}\right)+i \mathcal{Q} \bar{e} \bar{A}_{\mu}+i \bar{g}_{Z}\left(\tau_{3}-\sin ^{2} \theta_{Z} \mathcal{Q}\right) \bar{Z}_{\mu}
$$

where:

$$
\begin{aligned}
\bar{e} & =\bar{g}_{2}\left(\frac{1+c_{W^{ \pm}, \text {kin }}}{1+c_{W^{3}, \text { kin }}}\right)\left(\cos \bar{\theta} X_{12}+\sin \bar{\theta} X_{11}\right) \\
\bar{g}_{Z} & =\bar{g}_{2}\left(\frac{1+c_{W^{ \pm}, \mathrm{kin}}}{1+c_{W^{3}, \mathrm{kin}}}\right) \frac{\operatorname{det}(\mathrm{X})}{\cos \bar{\theta} X_{22}+\sin \bar{\theta} X_{21}} \\
\sin ^{2} \theta_{Z} & =-\frac{\left(\cos \bar{\theta} X_{12}+\sin \bar{\theta} X_{11}\right)\left(\cos \bar{\theta} X_{21}-\sin \bar{\theta} X_{22}\right)}{\operatorname{det}(\mathrm{X})} .
\end{aligned}
$$

The expanded couplings are:

$$
\begin{aligned}
\bar{e} & =\bar{g}_{2} \sin \bar{\theta}-\frac{v_{T}^{2} \bar{g}_{2}}{2 \Lambda^{2}} c_{H W B} \cos \bar{\theta} \\
& -\frac{v_{T}^{4} \bar{g}_{2}}{8 \Lambda^{4}}\left(2\left(c_{8, H W B}+2 c_{H W B}\left(c_{H W}+c_{H B}\right)\right) \cos \bar{\theta}-\left(4 c_{8, H W 2}+3 c_{H W B}^{2}\right) \sin \bar{\theta}\right) \\
\bar{g}_{Z} & =\frac{\bar{g}_{2}}{\cos \bar{\theta}}+\frac{v_{T}^{2} \bar{g}_{2}}{2 \Lambda^{2} \cos ^{2} \bar{\theta}} \\
& +\frac{v_{T}^{4} \bar{g}_{2}}{8 \Lambda^{4} \cos ^{3} \bar{\theta}}\left(2 \sin \bar{\theta} \cos \bar{\theta}\left(c_{8, H W B}+2 c_{H W B}\left(c_{H W}+c_{H B}\right)+\cos ^{2} \bar{\theta}\left(c_{H W B}^{2}+4 c_{8, H W 2}\right)\right)\right. \\
\sin ^{2} \theta_{Z} & =\sin ^{2} \bar{\theta}+\frac{v_{T}^{4}}{4 \Lambda^{4}} c_{H W B}^{2}\left(\sin ^{2} \bar{\theta}-\cos ^{2} \bar{\theta}\right) .
\end{aligned}
$$


The $\left(1+c_{W^{ \pm}, \text {kin }}\right) /\left(1+c_{W^{3} \text {,kin }}\right)$ factor in eq. (D.10) is due to the different normalizations of $W^{ \pm}$and $W^{3}$ and is $\propto c_{8, H W 2}$. As a result, $\sin ^{2} \theta_{Z} \neq \sin ^{2} \bar{\theta}$, meaning that the angle that rotates the gauge fields to mass eigenstates differs from the angle in the covariant derivative by $\mathcal{O}\left(1 / \Lambda^{4}\right){ }^{24}$

Lastly, we express the Higgs boson mass as:

$$
\begin{aligned}
M_{H}^{2}=2 \lambda v_{T}^{2} & \left(1-\frac{v_{T}^{2}}{2 \lambda \Lambda^{2}}\left(3 c_{H}+\lambda\left(c_{H D}-2 c_{H \square}\right)\right)-\frac{v_{T}^{4}}{4 \lambda \Lambda^{4}}\left(3 c_{H}\left(2 c_{H \square}-c_{H D}\right)\right.\right. \\
& \left.\left.+6 c_{8, H}+\lambda\left(c_{8, H D}+c_{8, H D 2}-\left(c_{H D}-2 c_{H \square}\right)^{2}\right)\right)\right)
\end{aligned}
$$

We are now ready to set the EW inputs. Following [73], it is convenient to write hatted quantities to represent those that are measured. Using $\left\{\hat{\alpha}_{e m}, \hat{M}_{Z}^{2}, \hat{G}_{F}, \hat{M}_{H}^{2}\right\}$, we can form the combinations:

$$
\begin{array}{lll}
\hat{e}=\sqrt{4 \pi \hat{\alpha}_{e m}}, & \hat{v}^{2}=\frac{1}{\sqrt{2} \hat{G}_{F}}, & \sin ^{2} \hat{\theta}=\frac{1}{2}\left(1-\sqrt{1-\frac{4 \pi \hat{\alpha}_{e m}}{\sqrt{2} \hat{G}_{F} \hat{M}_{Z}^{2}}}\right), \\
\hat{g}_{1}=\frac{\hat{e}}{c_{\hat{\theta}}}, & \hat{g}_{2}=\frac{\hat{e}}{s_{\hat{\theta}}}, & \hat{\lambda}=\frac{\hat{M}_{H}^{2}}{2 \hat{v}^{2}} .
\end{array}
$$

The task is now to solve for the barred input variables in terms of the hatted measured quantities, e.g. $\bar{g}_{1}\left(\hat{\alpha}_{e m}, \hat{M}_{Z}^{2}, \hat{G}_{F}\right)$, or $\bar{g}_{1}(\hat{e}, \hat{\theta}, \hat{v})$, with $\bar{g}_{1} \rightarrow \hat{g}_{1}$ in the limit that all higher dimension coefficients vanish.

The Fermi constant $\hat{G}_{F}$ is set by muon decay and determines $\hat{v}$ through eq. (D.13). In the SM, muon decay comes from $W$-boson exchange, so $\hat{G}_{F}$ is the ratio of the chargedcurrent (coupling) ${ }^{2}$ divided by the $W$ boson mass. The effects of higher dimensional operators are: i.) universal shifts to the charged-current coupling or $W$-boson mass, ii.) flavor-specific shifts in the charged current (e.g. shifts in $W$ boson coupling to $e \nu_{e}$ or $\mu \nu_{\mu}$ ), and iii.) 4-fermion contact terms. Calculated within the dimension-8 SMEFT, we find:

$$
\begin{aligned}
\frac{\hat{G}_{F}}{\sqrt{2}} & \stackrel{\operatorname{dim}-8}{=} \frac{\bar{g}_{2}^{2}}{8 m_{W}^{2}}+\frac{\delta G_{F 1}}{\Lambda^{2}}+\frac{v_{T}^{2} \delta G_{F 2}}{\Lambda^{4}} \\
& =\frac{1}{2 v_{T}^{2}}-\frac{v_{T}^{2}}{4 \sqrt{2} \Lambda^{4}}\left(c_{8, H D}-c_{8, H D 2}\right)+\frac{\delta G_{F 1}}{\Lambda^{2}}+\frac{v_{T}^{2} \delta G_{F 2}}{\Lambda^{4}}
\end{aligned}
$$

where we use $\delta G_{F 1}, \delta G_{F 2}$ to parametrize the non-universal contributions from fermionic dimension- 6 and dimension- 8 operators. If we maintain our restriction to $N_{f}=1$ fermion generation operators, then there are no four-fermion contributions to muon decay, as potential contributions like $\left(L_{2}^{\dagger} \bar{\sigma}^{\mu} \tau^{A} L_{2}\right)\left(L_{1}^{\dagger} \bar{\sigma}_{\mu} \tau^{A} L_{1}\right)$ necessarily involve multiple generations. At dimension-8, this restriction eliminates any operators of the form $\left(H^{\dagger} H\right)\left(L^{\dagger} L\right)^{2}$, as well

\footnotetext{
${ }^{24}$ The usual technique for coding kinetic terms into FeynRules assumes $\sin ^{2} \theta_{Z} \equiv \sin ^{2} \bar{\theta}$. One quick fix to compensate for the mismatch at dimension- 8 is to include new operators, e.g. $\bar{f} \gamma^{\mu} f Z_{\mu}$ with coefficient $\propto\left(\sin ^{2} \theta_{Z}-\sin ^{2} \bar{\theta}\right)$.
} 
as operators with the Higgs fields replaced by derivatives, ${ }^{25} D^{2}\left(L^{\dagger} L\right)^{2}$. Without the fourfermi contributions, only operators that shift the lepton- $W$ coupling contribute to $G_{F}$ : $\mathcal{O}_{H \ell}^{(3)}$ (in the notation of ref. [5]) at dimension-6, and the analog of table 7 operators with $Q \rightarrow L$ at dimension-8. While we leave the effects of $N_{f}>1$ on the interpretation of $G_{F}$ to future work, we can account for the shifts in the lepton- $W$ coupling as follows:

$$
\begin{aligned}
& \delta G_{F 1}=\sqrt{2} c_{H \ell}^{(3)} \\
& \delta G_{F 2}=\frac{1}{\sqrt{2}}\left(\left(c_{H \ell}^{(3)}\right)^{2}-\frac{1}{2}\left(2 c_{8, L 2}+c_{8, L 4}-i c_{8, L 3}\right)\right) .
\end{aligned}
$$

As expected, $\delta G_{F 2}$ receives contributions from $(\operatorname{dim}-6)^{2}$ and from (dim-8).

Inverting eq. (D.14) defines $v_{T}\left(\hat{v}, c_{i}\right)$ :

$$
v_{T}=\hat{v}\left(1+\frac{\hat{v}^{2}}{\Lambda^{2}} \delta G_{F 1}+\frac{\hat{v}^{4}}{8 \Lambda^{4}}\left(-c_{8, H}+c_{8, H D 2}+12 \delta G_{F 1}^{2}+8 \delta G_{F 2}\right)\right) .
$$

Having solved for $v_{T}$, we can set eqs. (D.10), (D.7) equal to the measured values $\hat{e}, \hat{M}_{Z}^{2}$, and then invert them to solve for $\bar{g}_{1}, \bar{g}_{2}$ (or some combination of them, such as $\sin \bar{\theta}, \cos \bar{\theta}$ ). Employing shorthand $\sin \hat{\theta}=s_{\hat{\theta}}, \cos \hat{\theta}=c_{\hat{\theta}}$, etc:

$$
\begin{aligned}
\bar{g}_{1}= & \frac{\hat{e}}{c_{\hat{\theta}}}\left(1+\frac{\hat{v}^{2} s_{\hat{\theta}}\left(4 c_{H W B} c_{\hat{\theta}}+\left(c_{H D}+4 \delta G_{F 1}\right) s_{\hat{\theta}}\right)}{4 \Lambda^{2} c_{2 \hat{\theta}}}-\frac{\hat{v}^{4}}{32 \Lambda^{4} c_{2 \hat{\theta}}^{3}} \times\left(-8 c_{8, H D 2} s_{\hat{\theta}}^{2} c_{2 \hat{\theta}}^{2}\right.\right. \\
& -8 c_{8, H W B} s_{2 \hat{\theta}} c_{2 \hat{\theta}}^{2}-4 c_{H W B}\left(c_{H W}+c_{H B}\right) \frac{s_{4 \hat{\theta}}^{2}}{s_{2 \hat{\theta}}^{2}}-4 c_{H W B}^{2}\left(6 c_{2 \hat{\theta}}+3 c_{4 \hat{\theta}}+7\right) s_{\hat{\theta}}^{2} \\
& +8 c_{H W B} c_{H D}\left(c_{2 \hat{\theta}}-2\right) s_{\hat{\theta}} c_{\hat{\theta}}^{3}-c_{H D}^{2}\left(5 c_{2 \hat{\theta}}+2\right) s_{\hat{\theta}}^{4}-2 \delta G_{F 1} c_{H W B}\left(11 s_{2 \hat{\theta}}+2 s_{4 \hat{\theta}}+3 s_{6 \hat{\theta}}\right) \\
& \left.\left.-2 \delta G_{F 1}\left(2 \delta G_{F 1}+c_{H D}\right)\left(6 c_{2 \hat{\theta}}+3 c_{4 \hat{\theta}}+7\right) s_{\hat{\theta}}^{2}-32 \delta G_{F 2} s_{\hat{\theta}}^{2} c_{2 \hat{\theta}}^{2}\right)\right) \\
\bar{g}_{2}= & \frac{\hat{e}}{s_{\hat{\theta}}}\left(1-\frac{\hat{v}^{2} c_{\hat{\theta}}\left(4 c_{H W B} s_{\hat{\theta}}+\left(c_{H D}+4 \delta G_{F 1}\right) c_{\hat{\theta}}\right)}{4 \Lambda^{2} c_{2 \hat{\theta}}}-\frac{\hat{v}^{4}}{32 \Lambda^{4} c_{2 \hat{\theta}}^{3}} \times\left(8 c_{8, H D 2} c_{\hat{\theta}}^{2} c_{2 \hat{\theta}}^{2}+16 c_{8, H W 2} c_{2 \hat{\theta}}^{3}\right.\right. \\
& +8 c_{8, H W B} s_{2 \hat{\theta}} c_{2 \hat{\theta}}^{2}+4 c_{H W B}\left(c_{H B}+c_{H W}\right) \frac{s_{4 \hat{\theta}}^{2}}{s_{2 \hat{\theta}}}+4 c_{H W B}^{2}\left(-6 c_{2 \hat{\theta}}-3 c_{4 \hat{\theta}}+7\right) c_{\hat{\theta}}^{2} \\
& +8 c_{H W B} c_{H D}\left(c_{2 \hat{\theta}}+2\right) s_{\hat{\theta}}^{3} c_{\hat{\theta}}-c_{H D}^{2}\left(5 c_{2 \hat{\theta}}-2\right) c_{\hat{\theta}}^{4}+2 \delta G_{F 1} c_{H W B}\left(11 s_{2 \hat{\theta}}-2 s_{4 \hat{\theta}}+3 s_{6 \hat{\theta}}\right) \\
& \left.\left.+2 \delta G_{F 1}\left(2 \delta G_{F 1}+c_{H D}\right)\left(-6 c_{2 \hat{\theta}}-3 c_{4 \hat{\theta}}+7\right) c_{\hat{\theta}}^{2}+32 \delta G_{F 2} c_{\hat{\theta}}^{2} c_{2 \hat{\theta}}^{2}\right)\right)
\end{aligned}
$$

Lastly, we can set eq. (D.12) equal to the measured Higgs mass $\hat{M}_{H}^{2}$ and invert to solve for the quartic coupling.

$$
\begin{gathered}
\lambda=\frac{\hat{M}_{H}^{2}}{2 \hat{v}^{2}}+\frac{1}{4 \Lambda^{2}}\left(\hat{M}_{H}^{2}\left(c_{H D}-4 c_{H \square}-4 \delta G_{F 1}\right)+6 c_{H} \hat{v}^{2}\right)+\frac{\hat{v}^{2}}{4 \Lambda^{4}}\left(\hat{M}_{H}^{2}\left(c_{8, H D}-4 \delta G_{F 2}\right)\right. \\
\left.+6\left(c_{8, H}+2 c_{H} \delta G_{F 1}\right) \hat{v}^{2}\right)
\end{gathered}
$$

With $\bar{g}_{1}, \bar{g}_{2}, v_{T}$ set, we can derive all other (EW) phenomenologically necessary parameters such as $m_{W}$ and $\sin ^{2} \theta_{Z}$.

\footnotetext{
${ }^{25}$ At dimension-8, only four-fermion operators with all left-handed fields are relevant.
} 


\section{E Explicit form for $\bar{q} q W, h W W$ and $\bar{q} q W h$ form factors}

The form factors for the $\bar{q} q W, h W W$ and $\bar{q} q W h$ vertices are listed below. In the following we have performed the field and coupling redefinitions following appendix D, but we have not re-expressed the couplings and vev in terms of measured EW inputs since that makes the expressions unwieldy.

$$
\begin{aligned}
c_{q q V 0}= & \frac{i \bar{g}_{2}}{\sqrt{2}}\left(1+\frac{v_{T}^{2}}{\Lambda^{2}} c_{H Q}^{(3)}-\frac{v_{T}^{4}}{4 \Lambda^{4}}\left(2 c_{8, Q 2}+c_{8, Q 4}-i c_{8, Q 3}\right)\right) \\
c_{q q V 1}= & \frac{i \bar{g}_{2} v_{T}^{2}}{2 \sqrt{2} \Lambda^{4}}\left(c_{8,3 Q 2}-c_{8,3 Q 4}\right) \\
c_{h V V 0}= & \frac{i \bar{g}_{2}^{2} v_{T}}{2}\left(1+\frac{v_{T}^{2}}{4 \Lambda^{2}}\left(4 c_{H \square}-c_{H D}\right)+\frac{v_{T}^{4}}{8 \Lambda^{4}}\left(5 c_{8, H D}-7 c_{8, H D 2}+\frac{3}{4}\left(c_{H D}-4 c_{H \square}\right)^{2}\right)\right) \\
c_{h V V 1}= & i\left(-\frac{4 v_{T}}{\Lambda^{2}} c_{H W}-\frac{v_{T}^{3}}{\Lambda^{4}}\left(4 c_{8, H W}-8 c_{H W}^{2}-c_{H W}\left(4 c_{H \square}-c_{H D}\right)\right)\right) \\
c_{h V V 2}= & \frac{-i \bar{g}_{2} v_{T}^{3}}{4 \Lambda^{4}}\left(c_{8, H D H W}-i c_{8, H D H W 2}\right) \\
c_{f f W h 0}= & i \sqrt{2} \bar{g}_{2} v_{T}\left(\frac{c_{H Q}^{(3)}}{\Lambda^{2}}-\frac{v_{T}^{2}}{4 \Lambda^{4}}\left(4 c_{8, Q 2}+2 c_{8, Q 4}-2 i c_{8, Q 3}-c_{H Q}^{(3)}\left(4 c_{H \square}-c_{H D}\right)\right)\right) \\
c_{f f W h 1}= & -i \frac{\bar{g}_{2} v_{T}}{\sqrt{2} \Lambda^{4}}\left(c_{8,3 Q 1}+c_{8,3 Q 3}\right) \\
c_{f f W h 2}= & -i \frac{\sqrt{2} \bar{g}_{2} v_{T}}{\Lambda^{4}}\left(c_{8, Q W 3}+i c_{8, Q W 5}\right) \\
c_{f f W h 3}= & i \frac{\bar{g}_{2} v_{T}}{\sqrt{2} \Lambda^{4}}\left(c_{8,3 Q 2}\right) \\
c_{f f W h 4}= & -i \frac{\bar{g}_{2} v_{T}}{\sqrt{2} \Lambda^{4}}\left(c_{8,3 Q 4}\right)
\end{aligned}
$$

\section{F Dimension-8 effects on other operators: $c_{H Q}^{(3)}$ and $c_{H \square}$}

In this appendix we repeat the analysis of section 4 in two other scenarios, one where the only non-zero dimension-6 operator is $\mathcal{O}_{H Q}^{(3)}$, and one with $\mathcal{O}_{H \square}$ only. In both cases, we set the dimension- 8 operator coefficients using the mixed-sign configuration of section 4 (i.e. all coefficients with equal magnitude, sign chosen to maximize effects at large $\sqrt{s}$ ). Expanding the partonic cross section $\hat{\sigma}\left(p p \rightarrow h W^{+}\right)$for large $\hat{s}$, these dimension- 6 operators contribute to different powers of $\hat{s}$ than $\mathcal{O}_{H W}$, so we expect the relative dimension- 8 effects to differ from $\mathcal{O}_{H W}$.

First we show the results for $\mathcal{O}_{H Q}^{(3)}$, whose coefficient $c_{H Q}^{(3)}$ modifies $\hat{\sigma}\left(p p \rightarrow h W^{+}\right)$at $\mathcal{O}(\hat{s})$. Comparing the domains of figure 4 with those of figures 2 and 3 we see that $c_{H Q}^{(3)}$ has a larger impact on $\sigma\left(p p \rightarrow h W^{+}\right)$than $c_{H W}$. This is not a surprise given that $c_{H Q}^{(3)}$ produces stronger $\hat{s}$ dependence. The dimension- 8 effects are still present. For example, at $\left|\Delta \mu\left(p p \rightarrow h W^{+}\right)\right|=0.2$, the variation between the scales inferred by the dimension- 6 only interpretation and the dimension- 6 plus maximum dimension- 8 interpretation is $3 \%$ 

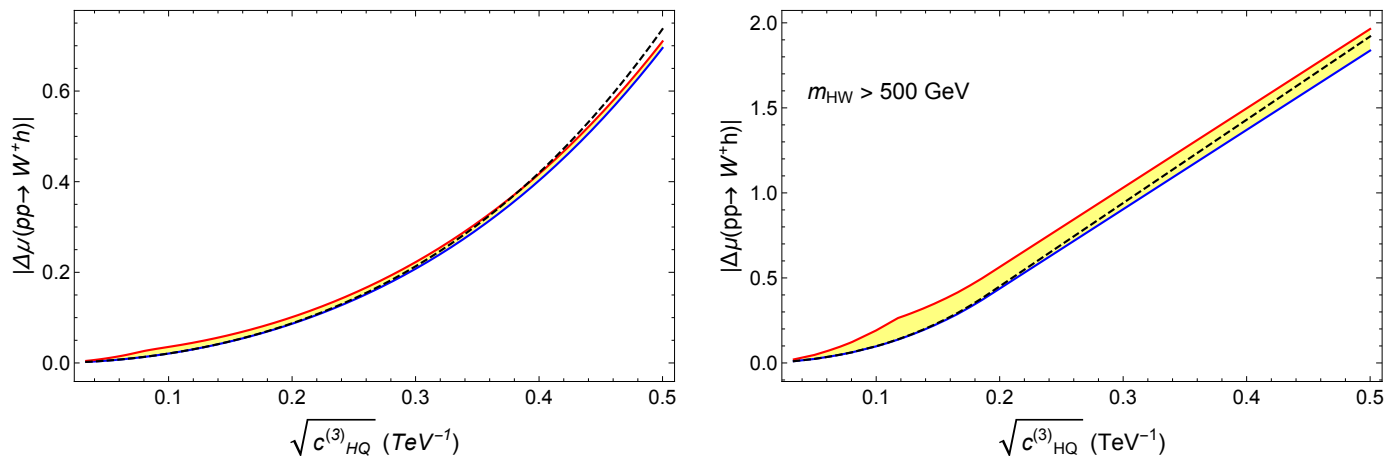

Figure 4. Deviation in the inclusive (left panel) and high-mass (right panel) $\sigma\left(p p \rightarrow h W^{+}\right.$) cross sections at the $\mathrm{LHC}(\sqrt{s}=13 \mathrm{TeV})$ assuming the only non-zero dimension-6 operator is $\mathcal{O}_{H Q}^{(3)}$ and adding in all dimension- 8 operators with equal magnitude coefficients and mixed signs as in the bottom panel of figure 2. The blue, red, and dashed black lines correspond to the same scenarios as in figure 2. The current limit on $c_{H Q}^{(3)}$ at $95 \% \mathrm{CL}$ is $0.66 \mathrm{TeV}^{-1}$ [90]; we have zoomed in to make the dimension- 8 contribution more visible.
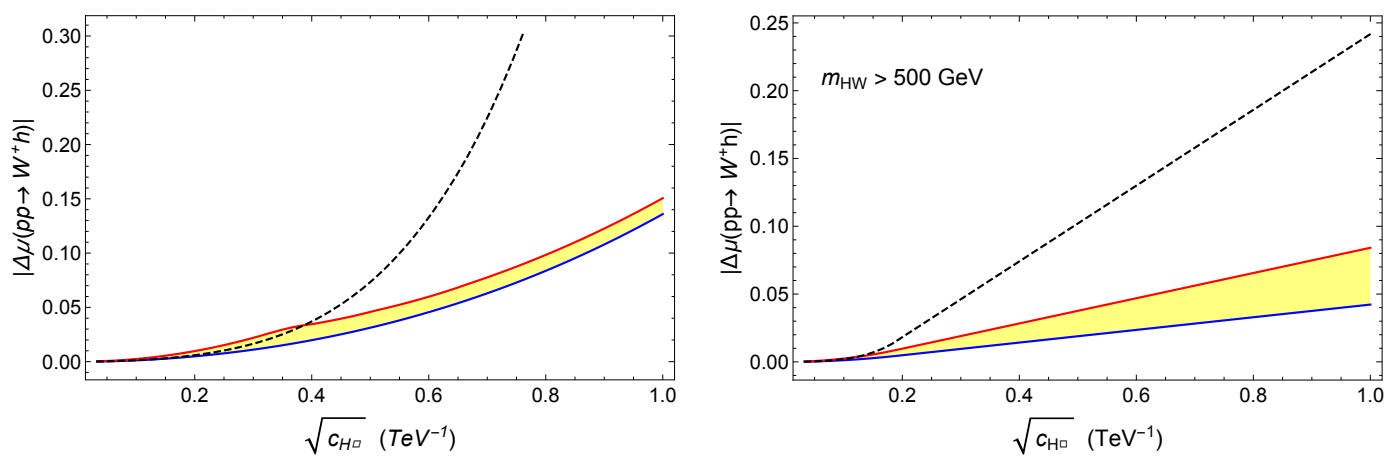

Figure 5. Deviation in the inclusive (left panel) and high-mass (right panel) $\sigma\left(p p \rightarrow h W^{+}\right)$cross sections at the LHC $(\sqrt{s}=13 \mathrm{TeV})$ assuming the only non-zero dimension-6 operator is $\mathcal{O}_{H \square}$ and adding in all dimension- 8 operators with equal magnitude coefficients and the mixed signs as in the bottom panel of figure 2. The blue, red, and dashed black lines correspond to the same scenarios as in figure 2. Current constraints on $c_{H \square}$ are weak, so we have zoomed in to make the dimension- 8 contribution more visible.

$\left(\Lambda_{N P}=3.39 \mathrm{TeV}\right.$ to $\left.\Lambda_{N P}=3.51 \mathrm{TeV}\right)$, while for $\left|\Delta \mu\left(p p \rightarrow h W^{+}\right)\right|_{m_{H W}>500 \mathrm{GeV}}=0.2$ the difference in scales increases to $37 \%\left(\Lambda_{N P}=7.12 \mathrm{TeV}\right.$ to $\left.\Lambda_{6}=9.79 \mathrm{TeV}\right)$. In both panels, the tapering effect at low values of the dimension- 6 coupling is less pronounced than it was in figures 2 and 3 . This is due to the fact that a larger dimension- 6 contribution to the cross section means $\Lambda_{8}$ can be lower before the $A_{S M} \times A_{\text {dim- } 6}>A_{S M} \times A_{\text {dim-8 }}$ EFT validity criteria is violated. The $\Lambda_{8}=\Lambda_{6}$ line in this scenario is difficult to see because it hugs the blue line.

Next, we repeat the exercise assuming the only dimension-6 operator is $\mathcal{O}_{H \square}$, with coefficient $c_{H \square}$. The results are shown in figure 5. As $c_{H \square}$ only modifies terms in the cross section at $\mathcal{O}\left(\hat{s}^{-1}\right)$, its impact on the cross section is very small. One might expect that a suppressed dimension- 6 piece could receive large relative dimension- 8 corrections. 
However, our EFT validity requirement $A_{S M} \times A_{\text {dim-6 }}>A_{S M} \times A_{\text {dim-8 }}$ prevents this from happening. Notice that the majority of the $\Lambda_{6}=\Lambda_{8}$ line lies outside of the region where we trust the EFT. In terms of new physics scales inferred by measurements of $|\Delta \mu|$, $\left|\Delta \mu\left(p p \rightarrow h W^{+}\right)\right|=0.1$, the new physics scale ranges from $1.15 \mathrm{TeV}$ (no dim-8) to $1.24 \mathrm{TeV}$ (max dim-8), a $7 \%$ shift.

Open Access. This article is distributed under the terms of the Creative Commons Attribution License (CC-BY 4.0), which permits any use, distribution and reproduction in any medium, provided the original author(s) and source are credited.

\section{References}

[1] S. Weinberg, Baryon and Lepton Nonconserving Processes, Phys. Rev. Lett. 43 (1979) 1566 [INSPIRE].

[2] W. Buchmüller and D. Wyler, Effective Lagrangian Analysis of New Interactions and Flavor Conservation, Nucl. Phys. B 268 (1986) 621 [INSPIRE].

[3] L. Lehman, Extending the Standard Model Effective Field Theory with the Complete Set of Dimension-7 Operators, Phys. Rev. D 90 (2014) 125023 [arXiv:1410.4193] [INSPIRE].

[4] B. Henning, X. Lu, T. Melia and H. Murayama, 2, 84, 30, 993, 560, 15456, 11962, 261485, ...: Higher dimension operators in the SM EFT, JHEP 08 (2017) 016 [arXiv:1512.03433] [INSPIRE].

[5] B. Grzadkowski, M. Iskrzynski, M. Misiak and J. Rosiek, Dimension-Six Terms in the Standard Model Lagrangian, JHEP 10 (2010) 085 [arXiv: 1008.4884] [INSPIRE].

[6] R. Contino, M. Ghezzi, C. Grojean, M. Muhlleitner and M. Spira, Effective Lagrangian for a light Higgs-like scalar, JHEP 07 (2013) 035 [arXiv: 1303.3876] [INSPIRE].

[7] R.S. Gupta, A. Pomarol and F. Riva, BSM Primary Effects, Phys. Rev. D 91 (2015) 035001 [arXiv: 1405.0181] [INSPIRE].

[8] E. Masso, An Effective Guide to Beyond the Standard Model Physics, JHEP 10 (2014) 128 [arXiv: 1406.6376] [INSPIRE].

[9] LHC Higgs Cross Section Working Group collaboration, Handbook of LHC Higgs Cross Sections: 4. Deciphering the Nature of the Higgs Sector, arXiv:1610.07922 [InSPIRE].

[10] A. Falkowski, B. Fuks, K. Mawatari, K. Mimasu, F. Riva and V. Sanz, Rosetta: an operator basis translator for Standard Model effective field theory, Eur. Phys. J. C 75 (2015) 583 [arXiv: 1508.05895] [INSPIRE].

[11] J. Aebischer et al., WCxf: an exchange format for Wilson coefficients beyond the Standard Model, Comput. Phys. Commun. 232 (2018) 71 [arXiv:1712.05298] [INSPIRE].

[12] Z. Han and W. Skiba, Effective theory analysis of precision electroweak data, Phys. Rev. D 71 (2005) 075009 [hep-ph/0412166] [INSPIRE].

[13] A. Pomarol and F. Riva, Towards the Ultimate SM Fit to Close in on Higgs Physics, JHEP 01 (2014) 151 [arXiv: 1308.2803] [INSPIRE].

[14] J. Ellis, V. Sanz and T. You, Complete Higgs Sector Constraints on Dimension-6 Operators, JHEP 07 (2014) 036 [arXiv:1404.3667] [INSPIRE]. 
[15] J. Ellis, V. Sanz and T. You, The Effective Standard Model after LHC Run I, JHEP 03 (2015) 157 [arXiv: 1410.7703] [INSPIRE].

[16] C.W. Murphy, Statistical approach to Higgs boson couplings in the standard model effective field theory, Phys. Rev. D 97 (2018) 015007 [arXiv:1710.02008] [INSPIRE].

[17] A. Falkowski and F. Riva, Model-independent precision constraints on dimension-6 operators, JHEP 02 (2015) 039 [arXiv: 1411.0669] [INSPIRE].

[18] A. Efrati, A. Falkowski and Y. Soreq, Electroweak constraints on flavorful effective theories, JHEP 07 (2015) 018 [arXiv:1503.07872] [INSPIRE].

[19] A. Falkowski, M. Gonzalez-Alonso, A. Greljo and D. Marzocca, Global constraints on anomalous triple gauge couplings in effective field theory approach, Phys. Rev. Lett. 116 (2016) 011801 [arXiv: 1508.00581] [INSPIRE].

[20] A. Falkowski and K. Mimouni, Model independent constraints on four-lepton operators, JHEP 02 (2016) 086 [arXiv: 1511.07434] [INSPIRE].

[21] A. Falkowski, M. Gonzalez-Alonso, A. Greljo, D. Marzocca and M. Son, Anomalous Triple Gauge Couplings in the Effective Field Theory Approach at the LHC, JHEP 02 (2017) 115 [arXiv: 1609.06312] [INSPIRE].

[22] A. Falkowski, M. González-Alonso and K. Mimouni, Compilation of low-energy constraints on 4-fermion operators in the SMEFT, JHEP 08 (2017) 123 [arXiv:1706. 03783] [INSPIRE].

[23] A. Falkowski, G. Grilli di Cortona and Z. Tabrizi, Future DUNE constraints on EFT, JHEP 04 (2018) 101 [arXiv: 1802.08296] [INSPIRE].

[24] T. Corbett, O.J.P. Eboli, J. Gonzalez-Fraile and M.C. Gonzalez-Garcia, Robust Determination of the Higgs Couplings: Power to the Data, Phys. Rev. D 87 (2013) 015022 [arXiv:1211.4580] [INSPIRE].

[25] T. Corbett, O.J.P. É boli, J. Gonzalez-Fraile and M.C. Gonzalez-Garcia, Determining Triple Gauge Boson Couplings from Higgs Data, Phys. Rev. Lett. 111 (2013) 011801 [arXiv:1304.1151] [INSPIRE].

[26] C. Englert, R. Kogler, H. Schulz and M. Spannowsky, Higgs coupling measurements at the LHC, Eur. Phys. J. C 76 (2016) 393 [arXiv:1511.05170] [inSPIRE].

[27] A. Buckley et al., Results from TopFitter, PoS (CKM2016) 127 (2016) [arXiv:1612.02294] [INSPIRE].

[28] A. Buckley et al., Constraining top quark effective theory in the LHC Run II era, JHEP 04 (2016) 015 [arXiv: 1512.03360] [INSPIRE].

[29] T. Corbett, O.J.P. Eboli, D. Goncalves, J. Gonzalez-Fraile, T. Plehn and M. Rauch, The Higgs Legacy of the LHC Run I, JHEP 08 (2015) 156 [arXiv:1505.05516] [INSPIRE].

[30] A. Butter, O.J.P. É boli, J. Gonzalez-Fraile, M.C. Gonzalez-Garcia, T. Plehn and M. Rauch, The Gauge-Higgs Legacy of the LHC Run I, JHEP 07 (2016) 152 [arXiv:1604.03105] [INSPIRE].

[31] B. Dumont, S. Fichet and G. von Gersdorff, A Bayesian view of the Higgs sector with higher dimensional operators, JHEP 07 (2013) 065 [arXiv: 1304.3369] [INSPIRE].

[32] J. de Blas et al., The Global Electroweak and Higgs Fits in the LHC era, PoS (EPS-HEP2017) 467 (2017) [arXiv: 1710.05402] [INSPIRE]. 
[33] J. de Blas et al., Electroweak precision constraints at present and future colliders, PoS (ICHEP2016) 690 (2017) [arXiv:1611.05354] [INSPIRE].

[34] L. Berthier and M. Trott, Towards consistent Electroweak Precision Data constraints in the SMEFT, JHEP 05 (2015) 024 [arXiv: 1502.02570] [INSPIRE].

[35] L. Berthier and M. Trott, Consistent constraints on the Standard Model Effective Field Theory, JHEP 02 (2016) 069 [arXiv:1508.05060] [INSPIRE].

[36] L. Berthier, M. Bjørn and M. Trott, Incorporating doubly resonant $W^{ \pm}$data in a global fit of SMEFT parameters to lift flat directions, JHEP 09 (2016) 157 [arXiv:1606.06693] [INSPIRE].

[37] I. Brivio and M. Trott, Scheming in the SMEFT... and a reparameterization invariance!, JHEP 07 (2017) 148 [arXiv: 1701.06424] [INSPIRE].

[38] M. Farina, G. Panico, D. Pappadopulo, J.T. Ruderman, R. Torre and A. Wulzer, Energy helps accuracy: electroweak precision tests at hadron colliders, Phys. Lett. B 772 (2017) 210 [arXiv: 1609.08157] [INSPIRE].

[39] S. Banerjee, C. Englert, R.S. Gupta and M. Spannowsky, Probing Electroweak Precision Physics via boosted Higgs-strahlung at the LHC, Phys. Rev. D 98 (2018) 095012 [arXiv: 1807.01796] [INSPIRE].

[40] C. Degrande, A basis of dimension-eight operators for anomalous neutral triple gauge boson interactions, JHEP 02 (2014) 101 [arXiv:1308.6323] [INSPIRE].

[41] D. Liu, A. Pomarol, R. Rattazzi and F. Riva, Patterns of Strong Coupling for LHC Searches, JHEP 11 (2016) 141 [arXiv:1603.03064] [INSPIRE].

[42] D. Liu and L.-T. Wang, Precision Measurement with Diboson at the LHC, arXiv: 1804.08688 [INSPIRE].

[43] A. Azatov, R. Contino, C.S. Machado and F. Riva, Helicity selection rules and noninterference for BSM amplitudes, Phys. Rev. D 95 (2017) 065014 [arXiv:1607.05236] [INSPIRE].

[44] L. Lehman and A. Martin, Hilbert Series for Constructing Lagrangians: expanding the phenomenologist's toolbox, Phys. Rev. D 91 (2015) 105014 [arXiv:1503. 07537] [InSPIRE].

[45] L. Lehman and A. Martin, Low-derivative operators of the Standard Model effective field theory via Hilbert series methods, JHEP 02 (2016) 081 [arXiv:1510.00372] [INSPIRE].

[46] P. Pouliot, Molien function for duality, JHEP 01 (1999) 021 [hep-th/9812015] [INSPIRE].

[47] S. Benvenuti, B. Feng, A. Hanany and Y.-H. He, Counting BPS Operators in Gauge Theories: Quivers, Syzygies and Plethystics, JHEP 11 (2007) 050 [hep-th/0608050] [INSPIRE].

[48] F.A. Dolan, Counting BPS operators in N=4 SYM, Nucl. Phys. B 790 (2008) 432 [arXiv: 0704.1038] [INSPIRE].

[49] J. Gray, A. Hanany, Y.-H. He, V. Jejjala and N. Mekareeya, SQCD: A Geometric Apercu, JHEP 05 (2008) 099 [arXiv: 0803.4257] [INSPIRE].

[50] A. Hanany, N. Mekareeya and G. Torri, The Hilbert Series of Adjoint SQCD, Nucl. Phys. B 825 (2010) 52 [arXiv: 0812.2315] [INSPIRE].

[51] Y. Chen and N. Mekareeya, The Hilbert series of U/SU SQCD and Toeplitz Determinants, Nucl. Phys. B 850 (2011) 553 [arXiv:1104.2045] [INSPIRE]. 
[52] A. Butti, D. Forcella, A. Hanany, D. Vegh and A. Zaffaroni, Counting Chiral Operators in Quiver Gauge Theories, JHEP 11 (2007) 092 [arXiv:0705.2771] [INSPIRE].

[53] B. Feng, A. Hanany and Y.-H. He, Counting gauge invariants: The Plethystic program, JHEP 03 (2007) 090 [hep-th/0701063] [INSPIRE].

[54] D. Forcella, A. Hanany and A. Zaffaroni, Baryonic Generating Functions, JHEP 12 (2007) 022 [hep-th/0701236] [inSPIRE].

[55] S. Benvenuti, A. Hanany and N. Mekareeya, The Hilbert Series of the One Instanton Moduli Space, JHEP 06 (2010) 100 [arXiv: 1005. 3026] [INSPIRE].

[56] A. Hanany, N. Mekareeya and S.S. Razamat, Hilbert Series for Moduli Spaces of Two Instantons, JHEP 01 (2013) 070 [arXiv:1205.4741] [INSPIRE].

[57] D. Rodríguez-Gómez and G. Zafrir, On the $5 d$ instanton index as a Hilbert series, Nucl. Phys. B 878 (2014) 1 [arXiv:1305.5684] [INSPIRE].

[58] A. Dey, A. Hanany, N. Mekareeya, D. Rodríguez-Gómez and R.-K. Seong, Hilbert Series for Moduli Spaces of Instantons on $\mathbb{C}^{2} / \mathbb{Z}_{n}$, JHEP 01 (2014) 182 [arXiv:1309.0812] [INSPIRE].

[59] A. Hanany and R.-K. Seong, Hilbert series and moduli spaces of $k \mathrm{U}(N)$ vortices, JHEP 02 (2015) 012 [arXiv: 1403.4950] [INSPIRE].

[60] L. Begin, C. Cummins and P. Mathieu, Generating functions for tensor products, hep-th/9811113 [INSPIRE].

[61] A. Hanany and R. Kalveks, Highest Weight Generating Functions for Hilbert Series, JHEP 10 (2014) 152 [arXiv: 1408.4690] [INSPIRE].

[62] B. Henning, X. Lu, T. Melia and H. Murayama, Operator bases, S-matrices and their partition functions, JHEP 10 (2017) 199 [arXiv:1706. 08520] [INSPIRE].

[63] E.E. Jenkins and A.V. Manohar, Algebraic Structure of Lepton and Quark Flavor Invariants and CP-violation, JHEP 10 (2009) 094 [arXiv:0907.4763] [INSPIRE].

[64] A. Hanany, E.E. Jenkins, A.V. Manohar and G. Torri, Hilbert Series for Flavor Invariants of the Standard Model, JHEP 03 (2011) 096 [arXiv: 1010.3161] [INSPIRE].

[65] A. Merle and R. Zwicky, Explicit and spontaneous breaking of $\mathrm{SU}(3)$ into its finite subgroups, JHEP 02 (2012) 128 [arXiv:1110.4891] [INSPIRE].

[66] A. Kobach and S. Pal, Hilbert Series and Operator Basis for NRQED and NRQCD/HQET, Phys. Lett. B 772 (2017) 225 [arXiv:1704.00008] [INSPIRE].

[67] A. Kobach and S. Pal, Conformal Structure of the Heavy Particle EFT Operator Basis, Phys. Lett. B 783 (2018) 311 [arXiv: 1804.01534] [INSPIRE].

[68] N.D. Christensen and C. Duhr, FeynRules - Feynman rules made easy, Comput. Phys. Commun. 180 (2009) 1614 [arXiv:0806.4194] [INSPIRE].

[69] C. Degrande, C. Duhr, B. Fuks, D. Grellscheid, O. Mattelaer and T. Reiter, UFO - The Universal FeynRules Output, Comput. Phys. Commun. 183 (2012) 1201 [arXiv:1108.2040] [INSPIRE].

[70] A. Alloul, N.D. Christensen, C. Degrande, C. Duhr and B. Fuks, FeynRules 2.0 - A complete toolbox for tree-level phenomenology, Comput. Phys. Commun. 185 (2014) 2250 [arXiv: 1310.1921] [INSPIRE]. 
[71] G. Isidori, A.V. Manohar and M. Trott, Probing the nature of the Higgs-like Boson via $h \rightarrow V \mathcal{F}$ decays, Phys. Lett. B 728 (2014) 131 [arXiv:1305.0663] [InSPIRE].

[72] G. Isidori and M. Trott, Higgs form factors in Associated Production, JHEP 02 (2014) 082 [arXiv: 1307.4051] [INSPIRE].

[73] R. Alonso, E.E. Jenkins, A.V. Manohar and M. Trott, Renormalization Group Evolution of the Standard Model Dimension Six Operators III: Gauge Coupling Dependence and Phenomenology, JHEP 04 (2014) 159 [arXiv:1312.2014] [INSPIRE].

[74] I. Brivio, Y. Jiang and M. Trott, The SMEFTsim package, theory and tools, JHEP 12 (2017) 070 [arXiv: 1709.06492] [INSPIRE].

[75] R.M. Fonseca, Calculating the renormalisation group equations of a SUSY model with Susyno, Comput. Phys. Commun. 183 (2012) 2298 [arXiv:1106.5016] [INSPIRE].

[76] H.K. Dreiner, H.E. Haber and S.P. Martin, Two-component spinor techniques and Feynman rules for quantum field theory and supersymmetry, Phys. Rept. 494 (2010) 1 [arXiv:0812.1594] [INSPIRE].

[77] E. Massa, Spinor equivalents of irreducible tensors under the special Lorentz group, Nuovo Cim. B 9 (1972) 41 [INSPIRE].

[78] B. Gripaios and D. Sutherland, DEFT: A program for operators in EFT, JHEP 01 (2019) 128 [arXiv: 1807.07546] [INSPIRE].

[79] ATLAS and CMS collaborations, Measurements of the Higgs boson production and decay rates and constraints on its couplings from a combined ATLAS and CMS analysis of the LHC pp collision data at $\sqrt{s}=7$ and $8 \mathrm{TeV}$, JHEP 08 (2016) 045 [arXiv:1606.02266] [INSPIRE].

[80] CMS collaboration, Evidence for the Higgs boson decay to a bottom quark-antiquark pair, Phys. Lett. B 780 (2018) 501 [arXiv: 1709.07497] [INSPIRE].

[81] CMS collaboration, Measurements of properties of the Higgs boson decaying to a $W$ boson pair in pp collisions at $\sqrt{s}=13 \mathrm{TeV}$, submitted to Phys. Lett. (2018) [arXiv:1806.05246] [INSPIRE].

[82] CMS collaboration, Measurements of Higgs boson properties in the diphoton decay channel in proton-proton collisions at $\sqrt{s}=13 \mathrm{TeV}$, JHEP 11 (2018) 185 [arXiv:1804.02716] [INSPIRE].

[83] ATLAS collaboration, Evidence for the $H \rightarrow b \bar{b}$ decay with the ATLAS detector, JHEP 12 (2017) 024 [arXiv: 1708. 03299] [INSPIRE].

[84] ATLAS collaboration, Measurements of the Higgs boson production cross section via Vector Boson Fusion and associated $W H$ production in the $W W^{*} \rightarrow \ell \nu \ell \nu$ decay mode with the ATLAS detector at $\sqrt{s}=13 \mathrm{TeV}$, ATLAS-CONF-2016-112.

[85] A. Alloul, B. Fuks and V. Sanz, Phenomenology of the Higgs Effective Lagrangian via FEYNRULES, JHEP 04 (2014) 110 [arXiv:1310.5150] [INSPIRE].

[86] C. Degrande, B. Fuks, K. Mawatari, K. Mimasu and V. Sanz, Electroweak Higgs boson production in the standard model effective field theory beyond leading order in QCD, Eur. Phys. J. C 77 (2017) 262 [arXiv:1609.04833] [inSPIRE].

[87] A. Adams, N. Arkani-Hamed, S. Dubovsky, A. Nicolis and R. Rattazzi, Causality, analyticity and an IR obstruction to UV completion, JHEP 10 (2006) 014 [hep-th/0602178] [INSPIRE]. 
[88] B. Bellazzini, L. Martucci and R. Torre, Symmetries, Sum Rules and Constraints on Effective Field Theories, JHEP 09 (2014) 100 [arXiv:1405.2960] [INSPIRE].

[89] A.D. Martin, W.J. Stirling, R.S. Thorne and G. Watt, Parton distributions for the LHC, Eur. Phys. J. C 63 (2009) 189 [arXiv:0901.0002] [inSPIRE].

[90] J. Ellis, C.W. Murphy, V. Sanz and T. You, Updated Global SMEFT Fit to Higgs, Diboson and Electroweak Data, JHEP 06 (2018) 146 [arXiv:1803.03252] [INSPIRE].

[91] ATLAS collaboration, Prospects for the study of the Higgs boson in the VH(bb) channel at HL-LHC, ATL-PHYS-PUB-2014-011.

[92] ATLAS collaboration, Projections for measurements of Higgs boson signal strengths and coupling parameters with the ATLAS detector at a HL-LHC, ATL-PHYS-PUB-2014-016.

[93] G.F. Giudice, C. Grojean, A. Pomarol and R. Rattazzi, The Strongly-Interacting Light Higgs, JHEP 06 (2007) 045 [hep-ph/0703164] [INSPIRE].

[94] CMS collaboration, Limits on anomalous triple and quartic gauge couplings, (2018) [https://twiki.cern.ch/twiki/bin/view/CMSPublic/PhysicsResultsSMPaTGC].

[95] ATLAS collaboration, Study of $W W \gamma$ and $W Z \gamma$ production in pp collisions at $\sqrt{s}=8 \mathrm{TeV}$ and search for anomalous quartic gauge couplings with the ATLAS experiment, Eur. Phys. J. C 77 (2017) 646 [arXiv:1707.05597] [INSPIRE].

[96] M.E. Peskin and D.V. Schroeder, An Introduction to quantum field theory, Addison-Wesley, Reading, U.S.A. (1995).

[97] B. Grinstein and M.B. Wise, Operator analysis for precision electroweak physics, Phys. Lett. B 265 (1991) 326 [INSPIRE]. 